An Efficient Approach to the Stereocontrolled Synthesis of Enamides.

Mathew V. J. Villa, Sarah M. Targett, John C. Barnes, William G. Whittingham, Rodolfo Marquez*

r.marquez@.chem.gla.ac.uk

Supporting Information.

Index

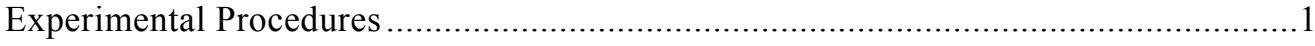

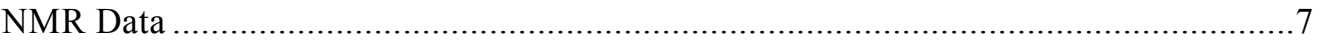


General Information. All reactions were performed in oven-dried glassware under an inert argon atmosphere unless otherwise stated. Anhydrous DMF was purchased from Aldrich Chemical Co. Tetrahydrofuran (THF), diethyl ether, and dichloromethane (DCM) were purified through a Pure Solv 400-5MD solvent purification system (Innovative Technology, Inc). All reagents were used as received, unless otherwise stated. Solvents were evaporated under reduced pressure at $40{ }^{\circ} \mathrm{C}$ using a Buchi Rotavapor.

IR spectra were recorded as thin films on $\mathrm{NaCl}$ plates using a Jasco FT/IR 4100 Fourier Transform spectrometer. Only significant absorptions (vmax) are reported in wavenumbers $\left(\mathrm{cm}^{-1}\right)$ with the following abbreviations used to describe absorption intensity: w, weak; m, medium; $\mathrm{s}$, strong and br, broad.

Proton magnetic resonance spectra $\left({ }^{1} \mathrm{H}-\mathrm{NMR}\right)$ were recorded at $400 \mathrm{MHz}$ using a Bruker DPX Avance400 instrument. Chemical shifts $\left(\delta_{\mathrm{H}}\right)$ are reported in parts per million (ppm), and are referenced to the residual solvent peak. The order of citation in parentheses is (1) number of equivalent nuclei (by integration), (2) multiplicity ( $\mathrm{s}=$ singlet, $\mathrm{d}=$ doublet, $\mathrm{t}=$ triplet, $\mathrm{q}=$ quartet, $\mathrm{qn}=$ quintet, $\mathrm{m}=$ multiplet, $\mathrm{br}=$ broad$)$, and (3) coupling constant $(\mathrm{J})$ quoted in Hertz to the nearest $0.5 \mathrm{~Hz}$. Carbon magnetic resonance spectra $\left({ }^{13} \mathrm{C}\right)$ were recorded at $100 \mathrm{MHz}$ using a Bruker DPX Avance400 instrument. Chemical shifts $\left(\delta_{\mathrm{C}}\right)$ are quoted in parts per million $(\mathrm{ppm})$ and are referenced to the appropriate solvent peak.

High resolution mass spectra were recorded on a JEOL JMS-700 spectrometer by electrospray ionisation mass spectrometer operating at a resolution of 15000 full widths at half height.

Flash chromatography was performed using silica gel (Apollo Scientific Silica Gel 60, 40-63 micron) as the stationary phase. TLC was performed on aluminium sheets pre-coated with silica (Merck Silica Gel $60 \mathrm{~F}_{254}$ ). The plates were visualised by the quenching of UV fluorescence $\left(\lambda_{\max } 254 \mathrm{~nm}\right)$ and/or by staining with either anisaldehyde, potassium permanganate or iodine followed by heating.

\section{Representative Procedures.}

General Procedure for the Synthesis of Lactam Derived $N$-Formyl Imides 9, 9a, 9b, 9c, 9d, and 9e. A mixture of formic acid $(0.1 \mathrm{~mol})$ and acetic acid $(0.1 \mathrm{~mol})$ was heated under argon at $55^{\circ} \mathrm{C}$ for 3 hours. The neat lactam $(\mathbf{8 - 8 e})(0.01 \mathrm{mmol})$ was then added, and the resulting reaction mixture stirred at $60{ }^{\circ} \mathrm{C}$ for 15 hours. The reaction solution was then cooled to room temperature and the excess mixed anhydride removed under vacuum (10 mbar). The resulting crude product was then suspended in ethyl acetate $(10 \mathrm{~mL})$ and the resulting solution filtered through a plug of neutral alumina to afford the desired lactam derived $n$-formyl imides $9,9 \mathbf{9}, \mathbf{9 b}, \mathbf{9 c}, \mathbf{9 d}$, and $9 \mathbf{e}$.

\section{2-Oxo-azetidine-1-carbaldehyde, 9a.}

${ }^{1} \mathrm{H}$ NMR $\left(400 \mathrm{MHz}, \mathrm{CDCl}_{3}\right) \delta 8.60(1 \mathrm{H}, \mathrm{s}), 3.60(2 \mathrm{H}, \mathrm{m}), 3.10(2 \mathrm{H}, \mathrm{m})$.

${ }^{13} \mathrm{C} \mathrm{NMR}\left(100 \mathrm{MHz}, \mathrm{CDCl}_{3}\right) \delta 166.0,156.3,22.1,20.5$. IR $v_{\max }(\mathrm{film}) / \mathrm{cm}^{-1} 2981(\mathrm{~s})$, 1830 (s), 1652(s). HRMS calcd for $\mathrm{C}_{4} \mathrm{H}_{5} \mathrm{O}_{2} \mathrm{~N}\left(\mathrm{M}^{+}\right)$: 99.0320. Found 99.0321.

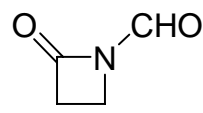

\section{2-Oxo-pyrrolidine-1-carbaldehyde, $9 \mathrm{~b}$.}

${ }^{1} \mathrm{H}$ NMR $\left(400 \mathrm{MHz}, \mathrm{CDCl}_{3}\right) \delta 9.01(1 \mathrm{H}, \mathrm{s}), 3.67(2 \mathrm{H}, \mathrm{td}, J=7.3,0.8 \mathrm{~Hz}), 2.54(2 \mathrm{H}, \mathrm{t}, J$ $=7.9 \mathrm{~Hz}), 2.07(2 \mathrm{H}, \mathrm{m}) .{ }^{13} \mathrm{C} \mathrm{NMR}\left(100 \mathrm{MHz}, \mathrm{CDCl}_{3}\right) \delta 176.8,160.3,42.1,32.2,17.8$. IR $v_{\max }\left(\right.$ film) $/ \mathrm{cm}^{-1} 2905$ (s), $1750(\mathrm{~s}), 1698(\mathrm{~s}), 1351$ (s), 1300 (m). HRMS calcd for $\mathrm{C}_{5} \mathrm{H}_{7} \mathrm{O}_{2} \mathrm{~N}\left(\mathrm{M}^{+}\right)$: 113.0477. Found 113.0479.

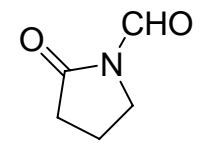

2-Oxo-piperidine-1-carbaldehyde, 9:

${ }^{1} \mathrm{H}$ NMR (400MHz, $\left.\mathrm{CDCl}_{3}\right)$ 8: $9.42(1 \mathrm{H}, \mathrm{s}), 3.55(2 \mathrm{H}, \mathrm{bs}), 2.55(2 \mathrm{H}, \mathrm{bm}), 1.80(4 \mathrm{H}$, bm). $\left.{ }^{13} \mathrm{C} \mathrm{NMR} \mathrm{(100MHz,} \mathrm{CDCl}_{3}\right) \delta: 173.2,162.8,41.6,33.3,21.7,19.9$.

IR $v_{\max }\left(\right.$ film) $/ \mathrm{cm}^{-1} 2958(\mathrm{~s}), 1690$ (s), 1717 (s). HRMS calcd for $\mathrm{C}_{6} \mathrm{H}_{10} \mathrm{O}_{2} \mathrm{~N}\left(\mathrm{M}+\mathrm{H}^{+}\right)$: 128.0712. Found 128.0710.

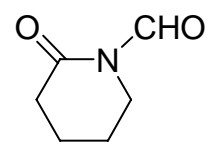


2-Oxo-azepane-1-carbaldehyde, 9c.

${ }^{1} \mathrm{H}$ NMR $\left(400 \mathrm{MHz}, \mathrm{CDCl}_{3}\right) \delta: 9.41(1 \mathrm{H}, \mathrm{s}), 3.81(2 \mathrm{H}, \mathrm{m}), 2.70(2 \mathrm{H}, \mathrm{m}), 1.81(4 \mathrm{H}, \mathrm{m})$, $1.71(2 \mathrm{H}, \mathrm{m}) .{ }^{13} \mathrm{C}$ NMR $\left(100 \mathrm{MHz}, \mathrm{CDCl}_{3}\right) \delta: 178.1,162.2,40.2,38.3,29.6,28.6,23.6$. IR $v_{\max }\left(\right.$ film) $/ \mathrm{cm}^{-1} 2986(\mathrm{~s}), 1700$ (s), 1695 (s). HRMS calcd for $\mathrm{C}_{7} \mathrm{H}_{12} \mathrm{O}_{2} \mathrm{~N}\left(\mathrm{M}+\mathrm{H}^{+}\right)$: 142.0868. Found 142.0870.

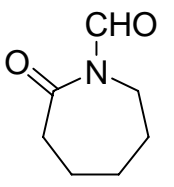

2-Oxo-azocane-1-carbaldehyde, 9d.

${ }^{1} \mathrm{H}$ NMR $\left(400 \mathrm{MHz}, \mathrm{CDCl}_{3}\right) \delta: 9.39(1 \mathrm{H}, \mathrm{s}), 3.77(2 \mathrm{H}, \mathrm{m}), 2.59(2 \mathrm{H}, \mathrm{m}), 1.85(2 \mathrm{H}, \mathrm{m})$, $1.65(2 \mathrm{H}, \mathrm{m}), 1.50(2 \mathrm{H}, \mathrm{m}), 1.45(2 \mathrm{H}, \mathrm{m}) .{ }^{13} \mathrm{C} \mathrm{NMR}\left(100 \mathrm{MHz}, \mathrm{CDCl}_{3}\right) \delta: 178.3,162.6$, 40.5, 35.0, 29.0, 28.6, 25.8, 24.3. IR $v_{\max }\left(\right.$ film) $/ \mathrm{cm}^{-1} 2935$ (s), 1652 (s), 1635 (s). HRMS calcd for $\mathrm{C}_{8} \mathrm{H}_{14} \mathrm{O}_{2} \mathrm{~N}\left(\mathrm{M}+\mathrm{H}^{+}\right)$: 156.1025. Found 156.1024.

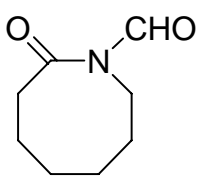

\section{2-Oxo-azonane-1-carbaldehyde, 9e.}

${ }^{1} \mathrm{H}$ NMR $\left(400 \mathrm{MHz}, \mathrm{CDCl}_{3}\right)$ 8: $9.35(1 \mathrm{H}, \mathrm{s}), 3.75(2 \mathrm{H}, \mathrm{m}), 2.61(2 \mathrm{H}, \mathrm{m}), 1.84(2 \mathrm{H}, \mathrm{m})$, $1.65(2 \mathrm{H}, \mathrm{m}), 1.55(2 \mathrm{H}, \mathrm{m}), 1.47(4 \mathrm{H}, \mathrm{m}) .{ }^{13} \mathrm{C} \mathrm{NMR}\left(100 \mathrm{MHz}, \mathrm{CDCl}_{3}\right) \delta: 178.8,162.9$, 42.3, 36.2, 28.1, 27.1, 25.1 (2C's), 22.5. IR $v_{\max }\left(\right.$ film) $/ \mathrm{cm}^{-1} 2932$ (s), 1695 (s), 1635 (s). HRMS calcd for $\mathrm{C}_{9} \mathrm{H}_{16} \mathrm{O}_{2} \mathrm{~N}\left(\mathrm{M}+\mathrm{H}^{+}\right)$: 170.1181. Found 170.1182 .

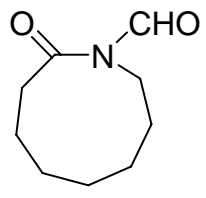

General Procedure for the Synthesis of Lactam Derived Enamides 10, 10a, 10b, 10c, 10d and 10e. A solution of the lactam derived $n$-formyl imide $(\mathbf{9 - 9 e})(1.0 \mathrm{mmol})$ in benzene $(10 \mathrm{~mL})$ was treated with ethoxycarbonylmethylene triphenylphosphorane $(3.0 \mathrm{mmol})$ and the resulting homogeneous mixture heated to $80^{\circ} \mathrm{C}$ for 18 hours. Once the reaction was complete by TLC analysis, the solvent was then removed in vacuo to generate a semi-solid crude residue. Purification of the crude product by flash column chromatography (silica gel, 30\% EtOAc in 40-60 petroleum ether) proceeded to generate the desired lactam derived E-enamides 10, 10a, 10b, 10c, 10d and 10e as a single double bond isomer in each case.

(E)-3-(2-Oxo-azetidin-1-yl)-acrylic acid ethyl ester, 10a.

${ }^{1} \mathrm{H}$ NMR $\left(400 \mathrm{MHz}, \mathrm{CDCl}_{3}\right) \delta: 7.65(1 \mathrm{H}, \mathrm{d}, J=14.0 \mathrm{~Hz}), 5.20(1 \mathrm{H}, \mathrm{d}, J=14.0$ $\mathrm{Hz}), 4.12(2 \mathrm{H}, \mathrm{q}, J=7.1 \mathrm{~Hz}), 3.45(2 \mathrm{H}, \mathrm{t}, J=4.9 \mathrm{~Hz}), 3.09(2 \mathrm{H}, \mathrm{t}, J=4.9 \mathrm{~Hz})$, $1.20(3 \mathrm{H}, \mathrm{t}, J=7.1 \mathrm{~Hz}) .{ }^{13} \mathrm{C} \mathrm{NMR}\left(100 \mathrm{MHz}, \mathrm{CDCl}_{3}\right) \delta: 166.9,164.9,134.9$, 100.0, 60.3, 38.8, 37.3, 14.3. IR $v_{\max }\left(\right.$ film) $/ \mathrm{cm}^{-1} 2983$ (s), 1774 (s), 1701 (s), 1632 (s), 1178 (s). HRMS calcd for $\mathrm{C}_{8} \mathrm{H}_{11} \mathrm{O}_{3} \mathrm{~N}\left(\mathrm{M}^{+}\right)$: 169.0739. Found 169.0738.

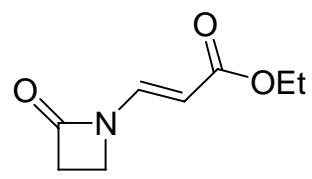

(E)-3-(2-Oxo-pyrrolidin-1-yl)-acrylic acid ethyl ester, $10 \mathrm{~b}$.

${ }^{1} \mathrm{H}$ NMR $\left(400 \mathrm{MHz}, \mathrm{CDCl}_{3}\right) \delta: 8.04(1 \mathrm{H}, \mathrm{d}, J=14.3 \mathrm{~Hz}), 5.14(1 \mathrm{H}, \mathrm{d}, J=14.2$ $\mathrm{Hz}), 4.13(2 \mathrm{H}, \mathrm{q}, J=7.1 \mathrm{~Hz}), 3.49(2 \mathrm{H}, \mathrm{t}, J=7.2 \mathrm{~Hz}), 2.49(2 \mathrm{H}, \mathrm{t}, J=7.9 \mathrm{~Hz})$, $2.1(2 \mathrm{H}$, appq, $J=7.5 \mathrm{~Hz}), 1.23(3 \mathrm{H}, \mathrm{t}, J=7.1 \mathrm{~Hz}) .{ }^{13} \mathrm{C} \mathrm{NMR}\left(100 \mathrm{MHz}, \mathrm{CDCl}_{3}\right)$ $\delta: 174.2,167.1,137.2,100.8,60.2,44.9,30.9,17.4,14.3$. IR $v_{\max }($ film $) / \mathrm{cm}^{-1}$ 2981 (s), 1728 (s), 1699 (s), 1630 (m), 1260 (m), 1159 (m). HRMS calcd for $\mathrm{C}_{9} \mathrm{H}_{13} \mathrm{O}_{3} \mathrm{~N}\left(\mathrm{M}^{+}\right)$: 183.0895 . Found 183.0888.

(E)-3-(2-Oxo-piperidin-1-yl)-acrylic acid ethyl ester, 10.

${ }^{1} \mathrm{H} \mathrm{NMR}\left(400 \mathrm{MHz}, \mathrm{CDCl}_{3}\right) \delta: 8.65(1 \mathrm{H}, \mathrm{d}, J=14.5 \mathrm{~Hz}), 5.30(1 \mathrm{H}, \mathrm{d}, J=14.5 \mathrm{~Hz})$, $4.25(2 \mathrm{H}, \mathrm{q}, J=7.1 \mathrm{~Hz}), 3.45(2 \mathrm{H}, \mathrm{t}, J=6.1 \mathrm{~Hz}), 2.60(2 \mathrm{H}, \mathrm{t}, J=6.7 \mathrm{~Hz}), 1.88$ $(2 \mathrm{H}, \mathrm{m}), 1.78(2 \mathrm{H}, \mathrm{m}), 1.30(3 \mathrm{H}, \mathrm{t}, J=7.1 \mathrm{~Hz}) \cdot{ }^{13} \mathrm{C} \mathrm{NMR}\left(100 \mathrm{MHz}, \mathrm{CDCl}_{3}\right) \delta$ : 169.5, 167.4, 140.7, 100.0, 60.1, 45.6, 33.1, 22.4, 20.2, 14.4. IR $v_{\max }(\mathrm{film}) / \mathrm{cm}^{-1}$ 2957 (s), 1708 (s), 1683 (s), 1622 (s), 1255 (s), 1159 (m). HRMS calcd for<smiles>CCOC(=O)/C=C/N1CCCC1=O</smiles>
$\mathrm{C}_{10} \mathrm{H}_{15} \mathrm{O}_{3} \mathrm{~N}\left(\mathrm{M}^{+}\right)$: 197.1052. Found 197.1050. 
(E)-3-(2-Oxo-azepan-1-yl)-acrylic acid ethyl ester, 10c.

${ }^{1} \mathrm{H}$ NMR $\left(400 \mathrm{MHz}, \mathrm{CDCl}_{3}\right) \delta: 8.39(1 \mathrm{H}, \mathrm{d}, J=14.5 \mathrm{~Hz}), 5.22(1 \mathrm{H}, \mathrm{d}, J=14.4$ $\mathrm{Hz}), 4.15(2 \mathrm{H}, \mathrm{q}, J=7.1 \mathrm{~Hz}), 3.55(2 \mathrm{H}, \mathrm{m}), 2.64(2 \mathrm{H}, \mathrm{m}), 1.70(6 \mathrm{H}, \mathrm{m}), 1.25$ $(3 \mathrm{H}, \mathrm{t}, J=7.06 \mathrm{~Hz}) .{ }^{13} \mathrm{C} \mathrm{NMR}\left(100 \mathrm{MHz}, \mathrm{CDCl}_{3}\right) \delta: 174.8,167.7,140.7,98.8$, 60.0, 45.4, 37.0, 29.1, 27.2, 23.4, 14.3. IR $v_{\max }\left(\right.$ film) $/ \mathrm{cm}^{-1} 2936$ (s), 1706 (s), 1684 (s), 1621 (s), 1173 (s), $1148(\mathrm{~m})$. HRMS calcd for $\mathrm{C}_{11} \mathrm{H}_{17} \mathrm{O}_{3} \mathrm{~N}\left(\mathrm{M}^{+}\right)$:<smiles>CCOC(=O)/C=C/N1CCCCCC1=O</smiles>
211.1208. Found 211.1207.

(E)-3-(2-Oxo-azocan-1-yl)-acrylic acid ethyl ester, 10d.

${ }^{1} \mathrm{H}$ NMR $\left(400 \mathrm{MHz}, \mathrm{CDCl}_{3}\right) \delta: 8.45(1 \mathrm{H}, \mathrm{d}, J=14.6 \mathrm{~Hz}), 5.25(1 \mathrm{H}, \mathrm{d}, J=14.6$ $\mathrm{Hz}), 4.15(2 \mathrm{H}, \mathrm{q}, J=7.1 \mathrm{~Hz}), 3.75(2 \mathrm{H}, \mathrm{m}), 2.60(2 \mathrm{H}, \mathrm{m}), 1.80(2 \mathrm{H}, \mathrm{m})$, 1.65 $(2 \mathrm{H}, \mathrm{m}), 1.50(2 \mathrm{H}, \mathrm{m}), 1.40(2 \mathrm{H}, \mathrm{m}), 1.22(3 \mathrm{H}, \mathrm{t}, J=7.1 \mathrm{~Hz}) .{ }^{13} \mathrm{C} \mathrm{NMR}$ $\left(100 \mathrm{MHz}, \mathrm{CDCl}_{3}\right) \delta: 174.6,167.6,139.6,100.2,60.1,44.0,34.5,29.0,27.2$, 26.1, 24.0, 14.4. IR $v_{\max }\left(\right.$ film) $/ \mathrm{cm}^{-1} 2935$ (s), 1702 (s), 1678 (s), 1621 (s), 1130

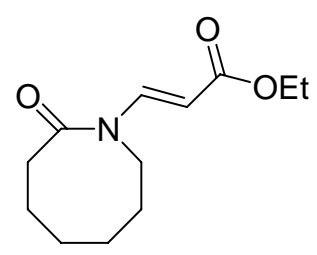
(s). HRMS calcd for $\mathrm{C}_{12} \mathrm{H}_{19} \mathrm{O}_{3} \mathrm{~N}\left(\mathrm{M}^{+}\right)$: 225.1365. Found 225.1364.

(E)-3-(2-Oxo-azonan-1-yl)-acrylic acid ethyl ester, 10e.

${ }^{1} \mathrm{H}$ NMR $\left(400 \mathrm{MHz}, \mathrm{CDCl}_{3}\right) \delta: 8.45(1 \mathrm{H}, \mathrm{d}, J=14.6 \mathrm{~Hz}), 5.21(1 \mathrm{H}, \mathrm{d}, J=14.6$ $\mathrm{Hz}), 4.14(2 \mathrm{H}, \mathrm{q}, J=7.1 \mathrm{~Hz}), 3.74(2 \mathrm{H}, \mathrm{m}), 2.50(2 \mathrm{H}, \mathrm{m}), 1.80(2 \mathrm{H}, \mathrm{m}), 1.70$ $(2 \mathrm{H}, \mathrm{m}), 1.50(2 \mathrm{H}, \mathrm{m}), 1.40(4 \mathrm{H}, \mathrm{m}), 1.25(3 \mathrm{H}, \mathrm{t}, J=7.1 \mathrm{~Hz}) .{ }^{13} \mathrm{C} \mathrm{NMR}$ $\left(100 \mathrm{MHz}, \mathrm{CDCl}_{3}\right) \delta: 175.5,167.6,140.0,100.3,60.1,45.7,35.2,28.1,25.3$, 25.2, 25.1, 22.2, 14.4. IR $v_{\max }\left(\right.$ film) $/ \mathrm{cm}^{-1} 2931$ (s), 1703 (s), 1676 (s), 1620 (s), $1155(\mathrm{~m})$. HRMS calcd for $\mathrm{C}_{13} \mathrm{H}_{21} \mathrm{O}_{3} \mathrm{~N}\left(\mathrm{M}^{+}\right)$: 239.1521. Found 239.1524 .

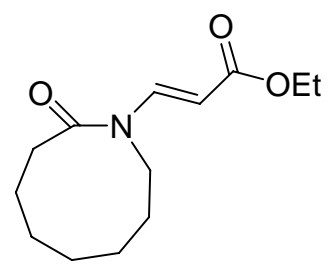

General Procedure for the Synthesis of $N$-Formyl Imides 12a, 12b, and 12c. A solution of the acyclic amide (11a-11c) $(1 \mathrm{mmol})$ in anhydrous THF $(10 \mathrm{~mL})$ was cooled to $0{ }^{\circ} \mathrm{C}$ before being treated with $n$-BuLi (1.1 mmol, 1.6 M solution in hexanes). The reaction was then stirred at $0{ }^{\circ} \mathrm{C}$ for 5 minutes before being treated with $\mathrm{N}$-formylbenzotriazole $(1.2 \mathrm{mmol}) .{ }^{1}$ The resulting mixture was then allowed to warm up to room temperature and then stirred for a further 2 hours. The reaction was diluted with $t$-butylmethyl ether $(10 \mathrm{~mL})$, and quenched with a saturated aq. $\mathrm{NaHCO}_{3}$ solution $(10$ $\mathrm{mL})$. The aqueous phase was then extracted with diethyl ether $(3 \times 20 \mathrm{~mL})$ and the combined organic layers dried over $\mathrm{Na}_{2} \mathrm{SO}_{4}$. The solvent was removed under vacuum to afford the crude product, which was then purified by flash column chromatography (silica gel, $10 \%$ to $20 \%$ ethyl acetate in $40-60$ petroleum ether) to afford the desired $N$-formyl imides (12a-12c).

\section{$N$-Formylbenzamide, 12a:}

${ }^{1} \mathrm{H}$ NMR (400MHz, $\left.\mathrm{CDCl}_{3}\right) \delta: 10.36(1 \mathrm{H}, \mathrm{bd}, J=7.7 \mathrm{~Hz}), 9.33(1 \mathrm{H}, \mathrm{d}, J=9.5$ $\mathrm{Hz}), 7.91(2 \mathrm{H}, \mathrm{m}), 7.56(1 \mathrm{H}, \mathrm{m}), 7.46(2 \mathrm{H}, \mathrm{m}) .{ }^{13} \mathrm{C} \mathrm{NMR}\left(100 \mathrm{MHz}, \mathrm{CDCl}_{3}\right) \delta$ : 165.8, 164.1, 132.9, 130.0, 128.0, 127.1. IR $v_{\max }($ film $) / \mathrm{cm}^{-1} 3414(\mathrm{~m}), 1728(\mathrm{~s})$, 1683 (s), 1463 (s), 1364 (m), 1252 (s), 1208 (s). HRMS calcd for $\mathrm{C}_{8} \mathrm{H}_{7} \mathrm{O}_{2} \mathrm{~N}$<smiles>O=CNC(=O)c1ccccc1</smiles>
$\left(\mathrm{M}^{+}\right)$: 149.0477. Found 149.0474.

\section{$N$-Formylnicotinamide, $12 b$.}

${ }^{1} \mathrm{H} \mathrm{NMR}\left(400 \mathrm{MHz}, \mathrm{CDCl}_{3}\right) \delta: 10.3(1 \mathrm{H}, \mathrm{bs}), 9.26(1 \mathrm{H}, \mathrm{d}, J=10.6 \mathrm{~Hz}), 8.58(1 \mathrm{H}$, $\mathrm{dm}, J=4.8 \mathrm{~Hz}), 8.19(1 \mathrm{H}, \mathrm{dm}, J=7.8 \mathrm{~Hz}), 7.89(1 \mathrm{H}, \mathrm{td}, J=7.7,1.7 \mathrm{~Hz}), 7.53$ $(1 \mathrm{H}, \mathrm{ddd}, J=7.6,4.7,1.1 \mathrm{~Hz}) .{ }^{13} \mathrm{C} \mathrm{NMR}\left(100 \mathrm{MHz}, \mathrm{CDCl}_{3}\right) \delta: 164.4,161.8$, 148.7, 147.1, 137.8, 128.1, 123.3. IR $v_{\max }(\mathrm{film}) / \mathrm{cm}^{-1} 3347$ (s), 2926 (s), 1736 (s),<smiles>O=CNC(=O)c1cccnc1</smiles>
1697 (s), 1462 (m), 1367 (s), $1183(\mathrm{~m})$. HRMS calcd for $\mathrm{C}_{7} \mathrm{H}_{6} \mathrm{O}_{2} \mathrm{~N}_{2}\left(\mathrm{M}^{+}\right)$: 150.0427. Found 150.0429.

\footnotetext{
${ }^{1}$ Katritzky, A. R.; Chang, H.-X.; Yang, B. Synthesis, 1995, 503.
} 
$N$-Formyl-(R)-2,2,5,5-tetramethyl-[1,3]dioxane-4-carboxylic acid amide, 12c. ${ }^{1} \mathrm{H}$ NMR $\left(400 \mathrm{MHz}, \mathrm{CDCl}_{3}\right) \delta: 9.08(1 \mathrm{H}, \mathrm{d}, J=10.4 \mathrm{~Hz}), 8.80(1 \mathrm{H}, \mathrm{bs}), 4.12(1 \mathrm{H}$, s), $3.65(1 \mathrm{H}, \mathrm{d}, J=11.8 \mathrm{~Hz}), 3.27(1 \mathrm{H}, \mathrm{d}, J=11.8 \mathrm{~Hz}), 1.41(3 \mathrm{H}, \mathrm{s}), 1.39(3 \mathrm{H}, \mathrm{s})$, $0.99(3 \mathrm{H}, \mathrm{s}), 0.98(3 \mathrm{H}, \mathrm{s}) .{ }^{13} \mathrm{C} \mathrm{NMR}\left(100 \mathrm{MHz}, \mathrm{CDCl}_{3}\right) \delta: 170.6,161.4,99.7$, 77.0, 71.1, 33.3, 29.3, 21.7, 18.9, 18.6. IR $v_{\max }\left(\right.$ film) $/ \mathrm{cm}^{-1} 3383$ (s), 2994 (s), 1744 (s), 1699 (s), 1459 (s), $1096(\mathrm{~m})$. HRMS calcd for $\mathrm{C}_{10} \mathrm{H}_{18} \mathrm{O}_{4} \mathrm{~N}\left(\mathrm{M}+\mathrm{H}^{+}\right)$:

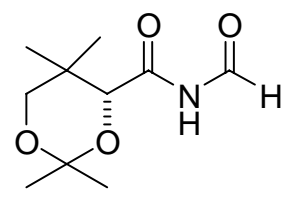
216.1236. Found 216.1233. $[\alpha]_{\mathrm{D}}+48.0\left(\mathrm{c}=1.4, \mathrm{CHCl}_{3}\right)$.

General Procedure for the Synthesis of Acyclic Enamides (13a-13c). A solution of $n$-formyl imide (12a-12c) $(1.0 \mathrm{mmol})$ in benzene $(10 \mathrm{~mL})$ was treated with ethoxycarbonylmethylene triphenylphosphorane $(3.0 \mathrm{mmol})$ and the resulting mixture heated to $95{ }^{\circ} \mathrm{C}$ for 19 hours. Upon reaction completion as indicated by TLC analysis, the solvent was removed under vacuum. The crude residue was then purified by flash column chromatography (silica gel, 10\% to 30\% EtOAc in 40-60 petroleum ether) to afford the desired enamides (13a-13c).

(Z)-3-Benzoylamino-acrylic acid ethyl ester, 13a (cis).

${ }^{1} \mathrm{H} \mathrm{NMR}\left(400 \mathrm{MHz}, \mathrm{CDCl}_{3}\right) \delta: 11.5(1 \mathrm{H}, \mathrm{bs}), 7.90(2 \mathrm{H}, \mathrm{m}), 7.68(1 \mathrm{H}, \mathrm{dd}, J=$ 9.1, $8.8 \mathrm{~Hz}), 7.53(1 \mathrm{H}, \mathrm{m}), 7.44(2 \mathrm{H}, \mathrm{m}), 5.20(1 \mathrm{H}, \mathrm{d}, J=8.8 \mathrm{~Hz}), 4.17(2 \mathrm{H}, \mathrm{q}$, $J=7.1 \mathrm{~Hz}), 1.27(3 \mathrm{H}, \mathrm{t}, J=7.1 \mathrm{~Hz}) .{ }^{13} \mathrm{C}$ NMR $\left(100 \mathrm{MHz}, \mathrm{CDCl}_{3}\right) \delta: 169.6$, 164.5, 138.8, 132.9, 132.2, 128.9, 127.7, 97.2, 60.3, 14.3. IR $v_{\max }($ film $) / \mathrm{cm}^{-1}$<smiles>CCOC(=O)/C=C\NC(=O)c1ccccc1</smiles>
3337 (s), 2985 (s), 1679 (s), 1627 (s), 1396 (m), 1381 (m), 1210 (m), 739 (s). HRMS calcd for $\mathrm{C}_{12} \mathrm{H}_{13} \mathrm{O}_{3} \mathrm{~N}\left(\mathrm{M}^{+}\right)$: 219. 0895. Found 219. 0898.

(E)-3-Benzoylamino-acrylic acid ethyl ester, 13a (trans).

${ }^{1} \mathrm{H}$ NMR $\left(400 \mathrm{MHz}, \mathrm{CDCl}_{3}\right) \delta: 8.28(1 \mathrm{H}, \mathrm{dd}, J=14.1,11.8 \mathrm{~Hz}), 8.08(1 \mathrm{H}$, bd, $J=11.8 \mathrm{~Hz}), 7.88(2 \mathrm{H}, \mathrm{m}), 7.64(1 \mathrm{H}, \mathrm{m}), 7.54(2 \mathrm{H}, \mathrm{m}), 5.65(1 \mathrm{H}, \mathrm{d}, J$ $=14.1 \mathrm{~Hz}), 4.25(2 \mathrm{H}, \mathrm{q}, J=7.1 \mathrm{~Hz}), 1.33(3 \mathrm{H}, \mathrm{t}, J=7.1 \mathrm{~Hz}) .{ }^{13} \mathrm{C} \mathrm{NMR}$ $\left(100 \mathrm{MHz}, \mathrm{CDCl}_{3}\right) \delta: 167.3,164.9,137.9,132.9,132.3,128.9,127.5$,<smiles>CCOC(=O)/C=C/NC(=O)c1ccccc1</smiles>
102.5, 60.3, 14.4. IR $v_{\max }\left(\right.$ film) $/ \mathrm{cm}^{-1} 3400$ (s), 2993 (s), 1694 (s), 1639 (s), 1095 (m), 1051 (m), 908 (s). HRMS calcd for $\mathrm{C}_{12} \mathrm{H}_{13} \mathrm{O}_{3} \mathrm{~N}\left(\mathrm{M}^{+}\right)$: 219.0895. Found 219.0897.

\section{(Z)-3-[(Pyridine-3-carbonyl)-amino]-acrylic acid ethyl ester, 13b (cis).}

${ }^{1} \mathrm{H} \mathrm{NMR}\left(400 \mathrm{MHz}, \mathrm{CDCl}_{3}\right) \delta: 12.35(1 \mathrm{H}, \mathrm{bs}), 8.67(1 \mathrm{H}, \mathrm{dm}, J=4.7 \mathrm{~Hz}), 8.18$ $(1 \mathrm{H}, \mathrm{dt}, J=7.8,1.0 \mathrm{~Hz}), 7.83(1 \mathrm{H}, \mathrm{td}, J=7.7,1.7 \mathrm{~Hz}), 7.60(1 \mathrm{H}, \mathrm{dd}, J=11.9$, $8.9 \mathrm{~Hz}), 7.44(1 \mathrm{H}, \mathrm{ddd}, J=7.6,4.7,1.2 \mathrm{~Hz}), 5.22(1 \mathrm{H}, \mathrm{d}, J=8.8 \mathrm{~Hz}), 4.21$ $(2 \mathrm{H}, \mathrm{q}, J=7.2 \mathrm{~Hz}), 1.28(3 \mathrm{H}, \mathrm{t}, J=7.17 \mathrm{~Hz}) .{ }^{13} \mathrm{C} \mathrm{NMR}\left(100 \mathrm{MHz}, \mathrm{CDCl}_{3}\right) \delta$ : 168.6, 162.9, 148.9, 148.5, 137.5, 137.0, 127.2, 123.3, 98.4, 60.3, 14.4. IR<smiles>CCOC(=O)/C=C\NC(=O)c1cccnc1</smiles>
$v_{\max }\left(\right.$ film) $/ \mathrm{cm}^{-1} 3424$ (s), 2985 (s), 1688 (s), 1625 (s), 1204 (m), 735 (s). HRMS calcd for $\mathrm{C}_{11} \mathrm{H}_{12} \mathrm{O}_{3} \mathrm{~N}_{2}\left(\mathrm{M}^{+}\right)$: 220.0848. Found 220. 0850 .

\section{(E)-3-[(Pyridine-3-carbonyl)-amino]-acrylic acid ethyl ester, 13b (trans).}

${ }^{1} \mathrm{H}$ NMR $\left(400 \mathrm{MHz}, \mathrm{CDCl}_{3}\right) \delta: 10.05(1 \mathrm{H}, \mathrm{bd}, J=10.4 \mathrm{~Hz}), 8.66(1 \mathrm{H}$, $\mathrm{dm}, J=4.8 \mathrm{~Hz}), 8.29(1 \mathrm{H}, \mathrm{dm}, J=7.8 \mathrm{~Hz}), 8.22(1 \mathrm{H}, \mathrm{dd}, J=14.2,12.2$ $\mathrm{Hz}), 7.95(1 \mathrm{H}, \mathrm{td}, J=7.7,1.7 \mathrm{~Hz}), 7.57(1 \mathrm{H}, \mathrm{ddd}, J=7.6,4.8,1.2 \mathrm{~Hz})$, $5.76(1 \mathrm{H}, \mathrm{d}, J=14.2 \mathrm{~Hz}), 4.26(2 \mathrm{H}, \mathrm{q}, J=7.1 \mathrm{~Hz}), 1.35(3 \mathrm{H}, \mathrm{t}, J=7.12$ $\mathrm{Hz}) .{ }^{13} \mathrm{C}$ NMR $\left(100 \mathrm{MHz}, \mathrm{CDCl}_{3}\right) \delta: 167.3,162.2,148.4,147.9,137.8$,<smiles>CCOC(=O)/C=C/NC(=O)c1cccnc1</smiles>
136.8, 127.39, 123.1, 103.3, 60.25, 14.40. IR $v_{\max }\left(\right.$ film) $/ \mathrm{cm}^{-1} 3274$ (s), 2971 (s), 1694 (s), 1637 (s), $1498(\mathrm{~s}), 1150(\mathrm{~m})$. HRMS calcd for $\mathrm{C}_{11} \mathrm{H}_{12} \mathrm{O}_{3} \mathrm{~N}_{2}\left(\mathrm{M}^{+}\right)$: 220.0848. Found 220.0847. 
(Z)-3-[((R)-2,2,5,5-Tetramethyl-[1,3]dioxane-4-carbonyl)-amino]-acrylic acid ethyl ester, 13c (cis).

${ }^{1} \mathrm{H}$ NMR $\left(400 \mathrm{MHz}, \mathrm{CDCl}_{3}\right) \delta: 11.05(1 \mathrm{H}, \mathrm{bs}), 7.36(1 \mathrm{H}, \mathrm{dd}, J=11.8,8.9$ $\mathrm{Hz}), 5.10(1 \mathrm{H}, \mathrm{d}, J=8.9 \mathrm{~Hz}), 4.14(1 \mathrm{H}, \mathrm{s}), 4.13(2 \mathrm{H}, \mathrm{q}, J=7.4 \mathrm{~Hz}), 3.66$ $(1 \mathrm{H}, \mathrm{d}, J=11.7 \mathrm{~Hz}), 3.26(1 \mathrm{H}, \mathrm{d}, J=11.7 \mathrm{~Hz}), 1.52(3 \mathrm{H}, \mathrm{s}), 1.40(3 \mathrm{H}, \mathrm{s})$, $1.23(3 \mathrm{H}, \mathrm{t}, J=7.2 \mathrm{~Hz}), 0.98(3 \mathrm{H}, \mathrm{s}), 0.97(3 \mathrm{H}, \mathrm{s}) .{ }^{13} \mathrm{C} \mathrm{NMR}(100 \mathrm{MHz}$,

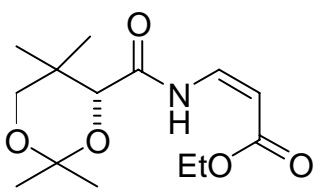
$\left.\mathrm{CDCl}_{3}\right) \delta: 168.7,168.2,135.9,99.3,98.1,76.7,71.3,60.1,33.3,29.3,21.9,19.0,18.6,14.3$. IR $v_{\max }\left(\right.$ film) $/ \mathrm{cm}^{-1} 3402$ (s), 2992 (s), 1690 (s), 1629 (s), 1197 (s), 731 (s). HRMS calcd for $\mathrm{C}_{14} \mathrm{H}_{23} \mathrm{O}_{5} \mathrm{~N}$ $\left(\mathrm{M}^{+}\right)$: 285.1576. Found 285.1574.

$(E)-3-[((R)-2,2,5,5-T e t r a m e t h y l-[1,3]$ dioxane-4-carbonyl)-amino]acrylic acid ethyl ester, 13c (trans).

${ }^{1} \mathrm{H}$ NMR $\left(400 \mathrm{MHz}, \mathrm{CDCl}_{3}\right) \delta: 8.35(1 \mathrm{H}, \mathrm{bd}, J=11.8 \mathrm{~Hz}), 7.91(1 \mathrm{H}, \mathrm{dd}$, $J=11.9,14.2 \mathrm{~Hz}), 5.25(1 \mathrm{H}, \mathrm{d}, J=14.2 \mathrm{~Hz}), 4.13(1 \mathrm{H}, \mathrm{s}), 4.12(2 \mathrm{H}, \mathrm{q}, J$ $=7.1 \mathrm{~Hz}), 3.65(1 \mathrm{H}, \mathrm{d}, J=11.8 \mathrm{~Hz}), 3.25(1 \mathrm{H}, \mathrm{d}, J=11.8 \mathrm{~Hz}), 1.44(3 \mathrm{H}$,

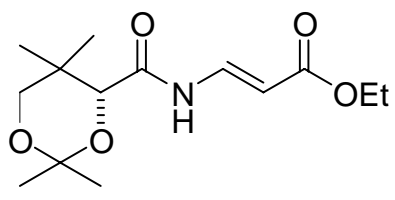
s), $1.38(3 \mathrm{H}, \mathrm{s}), 1.21(3 \mathrm{H}, \mathrm{t}, J=7.1 \mathrm{~Hz}), 0.98(3 \mathrm{H}, \mathrm{s}), 0.94(3 \mathrm{H}, \mathrm{s}) .{ }^{13} \mathrm{C} \mathrm{NMR}\left(100 \mathrm{MHz}, \mathrm{CDCl}_{3}\right) \delta$ : 166.9, 166.1, 134.8, 102.1, 98.5, 77.2, 70.2, 59.1, 32.3, 28.4, 20.8, 17.8, 17.6, 13.3. IR $v_{\max }($ film $) / \mathrm{cm}^{-1}$ 3399 (s), 2993 (s), 1716 (s), 1639 (s), 910 (s), 733 (m). HRMS calcd for $\mathrm{C}_{14} \mathrm{H}_{23} \mathrm{O}_{5} \mathrm{~N}\left(\mathrm{M}^{+}\right): 285.1576$. Found 285.1572. $[\alpha]_{\mathrm{D}}+45.6\left(\mathrm{c}=0.5, \mathrm{CHCl}_{3}\right)$.

General Procedure for the Synthesis of Acyclic Methyl-Enamides (14a-14c). A solution of $n$ formyl imide (12a-12c) $(1.0 \mathrm{mmol})$ in benzene $(10 \mathrm{~mL})$ was treated with 1-carbethoxyethylidene triphenylphosphorane $(3.0 \mathrm{mmol})$ and the resulting mixture heated to $95{ }^{\circ} \mathrm{C}$ for 19 hours. Upon reaction completion as indicated by TLC analysis, the solvent was removed under vacuum. The crude residue was then purified by flash column chromatography (silica gel, 10\% to 30\% EtOAc in 40-60 petroleum ether) to afford the desired enamides $16 \mathbf{c}, \mathbf{1 7 c}$ and $\mathbf{1 8 c}$.

(Z)-3-Benzoylamino-2-methyl-acrylic acid ethyl ester, 14a (cis).

${ }^{1} \mathrm{H}$ NMR $\left(400 \mathrm{MHz}, \mathrm{CDCl}_{3}\right) \delta: 11.40(1 \mathrm{H}, \mathrm{d}, J=10.8 \mathrm{~Hz}), 7.87(2 \mathrm{H}, \mathrm{m}), 7.56$ $(1 \mathrm{H}, \mathrm{dd}, J=10.9,1.2 \mathrm{~Hz}), 7.49(1 \mathrm{H}, \mathrm{m}), 7.42(2 \mathrm{H}, \mathrm{m}), 4.20(2 \mathrm{H}, \mathrm{q}, J=7.1$ $\mathrm{Hz}), 1.85(3 \mathrm{H}, \mathrm{d}, J=1.3 \mathrm{~Hz}), 1.29(3 \mathrm{H}, \mathrm{t}, J=7.1 \mathrm{~Hz}) .{ }^{13} \mathrm{C} \mathrm{NMR}(100 \mathrm{MHz}$, $\left.\mathrm{CDCl}_{3}\right) \delta: 169.0,168.1,134.3 .132 .3,131.6,127.8,126.5,104.6,59.6,16.3$,<smiles>CCOC(=O)/C(C)=C\NC(=O)c1ccccc1</smiles>
13.2.

(E)-3-Benzoylamino-2-methyl-acrylic acid ethyl ester, 14a (trans). ${ }^{1} \mathrm{H}$ NMR $\left(400 \mathrm{MHz}, \mathrm{CDCl}_{3}\right) \delta: 8.19(1 \mathrm{H}, \mathrm{bd}, J=11.7 \mathrm{~Hz}), 8.10(1 \mathrm{H}, \mathrm{dd}$, $J=11.6,1.2 \mathrm{~Hz}), 7.73(2 \mathrm{H}, \mathrm{m}), 7.45(1 \mathrm{H}, \mathrm{m}), 7.34(2 \mathrm{H}, \mathrm{m}), 4.09(2 \mathrm{H}, \mathrm{q}$, $J=7.14 \mathrm{~Hz}), 1.82(3 \mathrm{H}, \mathrm{d}, J=1.3 \mathrm{~Hz}), 1.20(3 \mathrm{H}, \mathrm{t}, J=7.1 \mathrm{~Hz}) .{ }^{13} \mathrm{C}$<smiles>CCOC(=O)/C(C)=C/NC(=O)c1ccccc1</smiles>
NMR $\left(100 \mathrm{MHz}, \mathrm{CDCl}_{3}\right) \delta: 168.1,164.5,132.8,132.7,131.9,128.9,127.3,109.0,60.6,14.4,10.8$. IR $v_{\max }\left(\right.$ film) $/ \mathrm{cm}^{-1} 3335$ (s), 2983 (s), 1684 (s), 1649 (s), 1267 (m), 1133 (s). HRMS calcd for $\mathrm{C}_{13} \mathrm{H}_{15} \mathrm{O}_{3} \mathrm{~N}$ $\left(\mathrm{M}^{+}\right)$: 233.1052. Found 233.1053.

(Z)-2-Methyl-3-[(pyridine-3-carbonyl)-amino]-acrylic acid ethyl ester, 14b (cis).

${ }^{1} \mathrm{H}$ NMR $\left(400 \mathrm{MHz}, \mathrm{CDCl}_{3}\right) \delta: 8.64(1 \mathrm{H}, \mathrm{dm}, J=4.8 \mathrm{~Hz}), 8.17(1 \mathrm{H}, \mathrm{dt}, J=$ $7.8,1.1 \mathrm{~Hz}), 7.82(1 \mathrm{H}, \mathrm{td}, J=7.7,1.7 \mathrm{~Hz}), 7.49(1 \mathrm{H}, \mathrm{dq}, J=11.8,1.3 \mathrm{~Hz})$, $7.41(1 \mathrm{H}, \mathrm{ddd}, J=7.6,4.7,1.1 \mathrm{~Hz}), 4.25(2 \mathrm{H}, \mathrm{q}, J=7.2 \mathrm{~Hz}), 1.87(3 \mathrm{H}, \mathrm{d}, J=$<smiles>CCOC(=O)/C(C)=C\NC(=O)c1cccnc1</smiles>
$1.3 \mathrm{~Hz}), 1.20(3 \mathrm{H}, \mathrm{t}, J=7.1 \mathrm{~Hz}) .{ }^{13} \mathrm{C} \mathrm{NMR}\left(100 \mathrm{MHz}, \mathrm{CDCl}_{3}\right) \delta: 167.9,161.3,147.9,147.8,136.3$, 132.4, 125.8, 122.1, 105.9, 59.5, 15.5, 13.3. IR $v_{\max }\left(\right.$ film) $/ \mathrm{cm}^{-1} 3391$ (s), 2963 (s), 1694 (s), 1632 (s), 996 (s). HRMS calcd for $\mathrm{C}_{12} \mathrm{H}_{14} \mathrm{O}_{3} \mathrm{~N}_{2}\left(\mathrm{M}^{+}\right)$: 234.1004. Found 234.1007. 
(E)-2-Methyl-3-[(pyridine-3-carbonyl)-amino]-acrylic acid ethyl ester, 14b (trans).

${ }^{1} \mathrm{H} \mathrm{NMR}\left(400 \mathrm{MHz}, \mathrm{CDCl}_{3}\right) \delta: 9.87(1 \mathrm{H}, \mathrm{d}, J=12.4 \mathrm{~Hz}), 8.52(1 \mathrm{H}, \mathrm{dm}, J$ $=4.8 \mathrm{~Hz}), 8.14(1 \mathrm{H}, \mathrm{dm}, J=7.8 \mathrm{~Hz}), 8.06(1 \mathrm{H}, \mathrm{dq} J=12.5,1.3 \mathrm{~Hz}), 7.82$ $(1 \mathrm{H}, \mathrm{td}, J=7.7,1.6 \mathrm{~Hz}), 7.45(1 \mathrm{H}$, ddd, $J=7.6,4.7,1.2 \mathrm{~Hz}), 4.15(2 \mathrm{H}$,<smiles>CCOC(=O)/C(C)=C/NC(=O)c1cccnc1</smiles>
q, $J=7.1 \mathrm{~Hz}), 1.89(3 \mathrm{H}, \mathrm{d}, J=1.4 \mathrm{~Hz}), 1.22(3 \mathrm{H}, \mathrm{t}, J=7.1 \mathrm{~Hz}) .{ }^{13} \mathrm{C} \mathrm{NMR}\left(100 \mathrm{MHz}, \mathrm{CDCl}_{3}\right) \delta$ : 168.2, 161.7, 148.4, 148.3, 137.7, 131.05, 127.2, 123.0, 109.9, 60.52, 14.43, 10.84. IR $v_{\max }(\mathrm{film}) / \mathrm{cm}^{-1}$ 3367 (s), 2986 (s), 1692 (s), 1652 (s), 1498 (s), 1265 (m). HRMS calcd for $\mathrm{C}_{12} \mathrm{H}_{14} \mathrm{O}_{3} \mathrm{~N}_{2}\left(\mathrm{M}^{+}\right)$: 234.1004. Found 234.1006.

(Z)-2-Methyl-3-[((R)-2,2,5,5-tetramethyl-[1,3]dioxane-4-carbonyl)amino]-acrylic acid ethyl ester, 14c (cis).

${ }^{1} \mathrm{H}$ NMR $\left(400 \mathrm{MHz}, \mathrm{CDCl}_{3}\right) \delta: 10.9(1 \mathrm{H}, \mathrm{bd}, J=12.4 \mathrm{~Hz}), 7.26(1 \mathrm{H}, \mathrm{dd}, J=$ $11.5,1.3 \mathrm{~Hz}), 4.18(2 \mathrm{H}, \mathrm{qd}, J=7.2,1.4 \mathrm{~Hz}), 4.13(1 \mathrm{H}, \mathrm{s}), 3.66(1 \mathrm{H}, \mathrm{d}, J=$ $11.7 \mathrm{~Hz}), 3.26(1 \mathrm{H}, \mathrm{d}, J=11.7 \mathrm{~Hz}), 1.79(3 \mathrm{H}, \mathrm{d}, J=1.3 \mathrm{~Hz}), 1.51(3 \mathrm{H}, \mathrm{s})$,

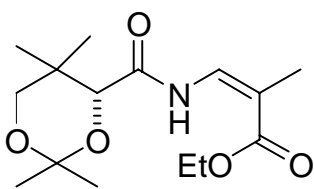
$1.39(3 \mathrm{H}, \mathrm{s}), 1.25(3 \mathrm{H}, \mathrm{t}, J=7.1 \mathrm{~Hz}), 0.98(3 \mathrm{H}, \mathrm{s}), 0.96(3 \mathrm{H}, \mathrm{s}) .{ }^{13} \mathrm{C} \mathrm{NMR}\left(100 \mathrm{MHz}, \mathrm{CDCl}_{3}\right) \delta: 167.6$, $167.3,131.3,105.5,98.2,75.7,70.4,60.1,32.2,28.4,20.9,18.0,17.6,15.4,13.4$.

(E)-2-Methyl-3-[((R)-2,2,5,5-tetramethyl-[1,3]dioxane-4-carbonyl)amino]-acrylic acid ethyl ester, 14c (trans).

${ }^{1} \mathrm{H}$ NMR $\left(400 \mathrm{MHz}, \mathrm{CDCl}_{3}\right) \delta: 8.25(1 \mathrm{H}, \mathrm{bd}, J=12.3), 7.86(1 \mathrm{H}, \mathrm{dq}, J$ $=12.3,1.4 \mathrm{~Hz}), 4.14(1 \mathrm{H}, \mathrm{s}), 4.10(2 \mathrm{H}, \mathrm{qd}, J=7.2,1.2 \mathrm{~Hz}), 3.64(1 \mathrm{H}$, d, $J=11.8 \mathrm{~Hz}), 3.22(1 \mathrm{H}, \mathrm{d}, J=11.8 \mathrm{~Hz}), 1.71(3 \mathrm{H}, \mathrm{d}, J=1.4 \mathrm{~Hz})$, $1.41(3 \mathrm{H}, \mathrm{s}), 1.39(3 \mathrm{H}, \mathrm{s}), 1.25(3 \mathrm{H}, \mathrm{t}, J=7.1 \mathrm{~Hz}), 0.97(3 \mathrm{H}, \mathrm{s}), 0.91(3 \mathrm{H}, \mathrm{s}) .{ }^{13} \mathrm{C}$ NMR $(100 \mathrm{MHz}$, $\left.\mathrm{CDCl}_{3}\right)$ 8: 168.0, 167.4, 130.1, 109.2, 99.3, 77.2, 71.2, 60.5, 33.2, 29.4, 21.8, 18.8, 18.6, 14.38, 10.4. IR $v_{\max }\left(\right.$ film) $/ \mathrm{cm}^{-1} 3410$ (s), 2992 (s), 1695 (s), 1652 (s). HRMS calcd for $\mathrm{C}_{15} \mathrm{H}_{25} \mathrm{O}_{5} \mathrm{~N}\left(\mathrm{M}^{+}\right): 299.1733$. Found 1735. $[\alpha]_{\mathrm{D}}+40.7\left(\mathrm{c}=1.1, \mathrm{CHCl}_{3}\right)$. 
2-Oxo-azetidine-1-carbaldehyde, 9a.

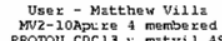

$\stackrel{7}{+\infty}$
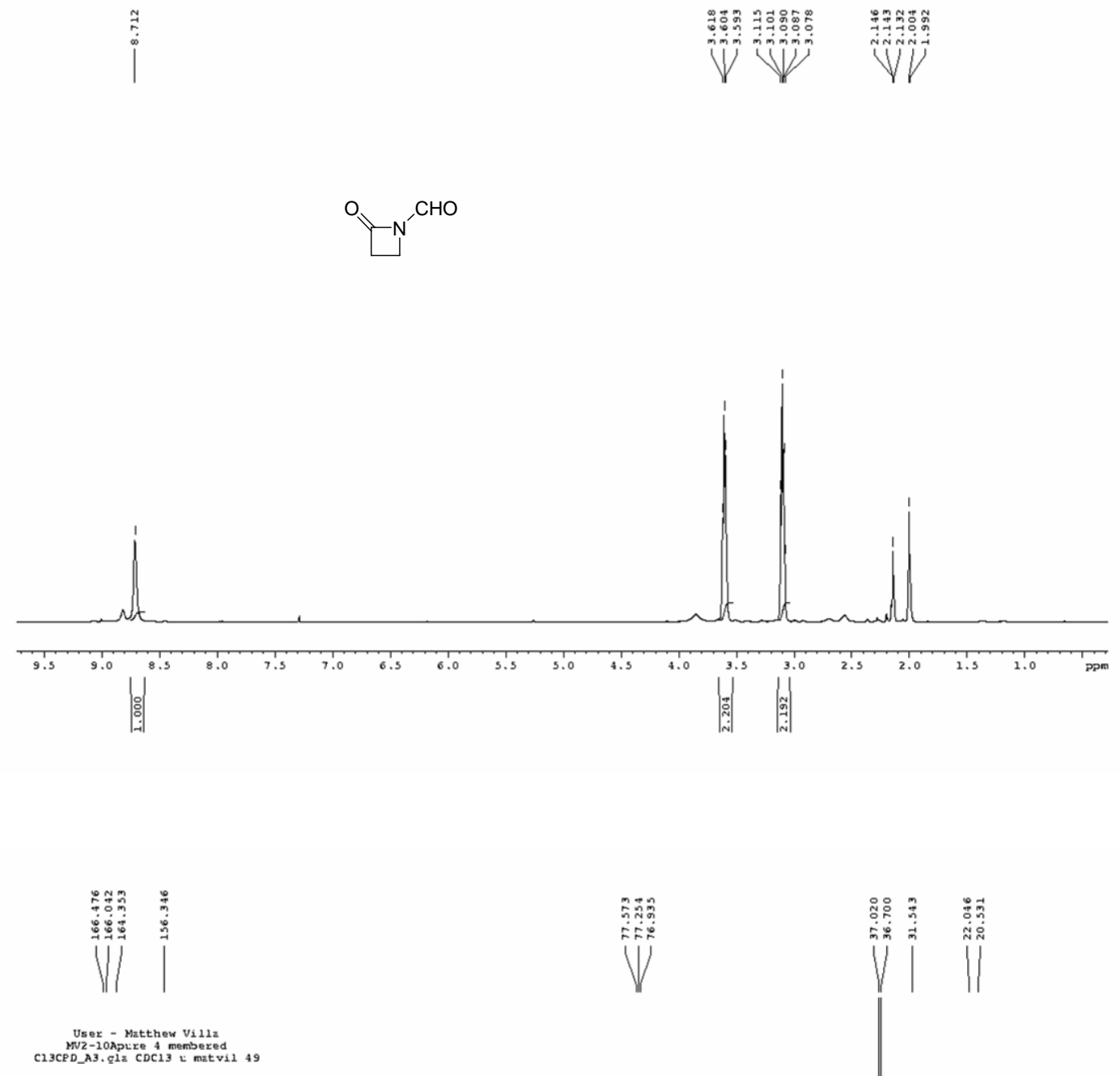

User - Matthew Villa MN2-10Aptre 4 membered
C13CPD_A3. Q12 CDC13 5 matvil 49
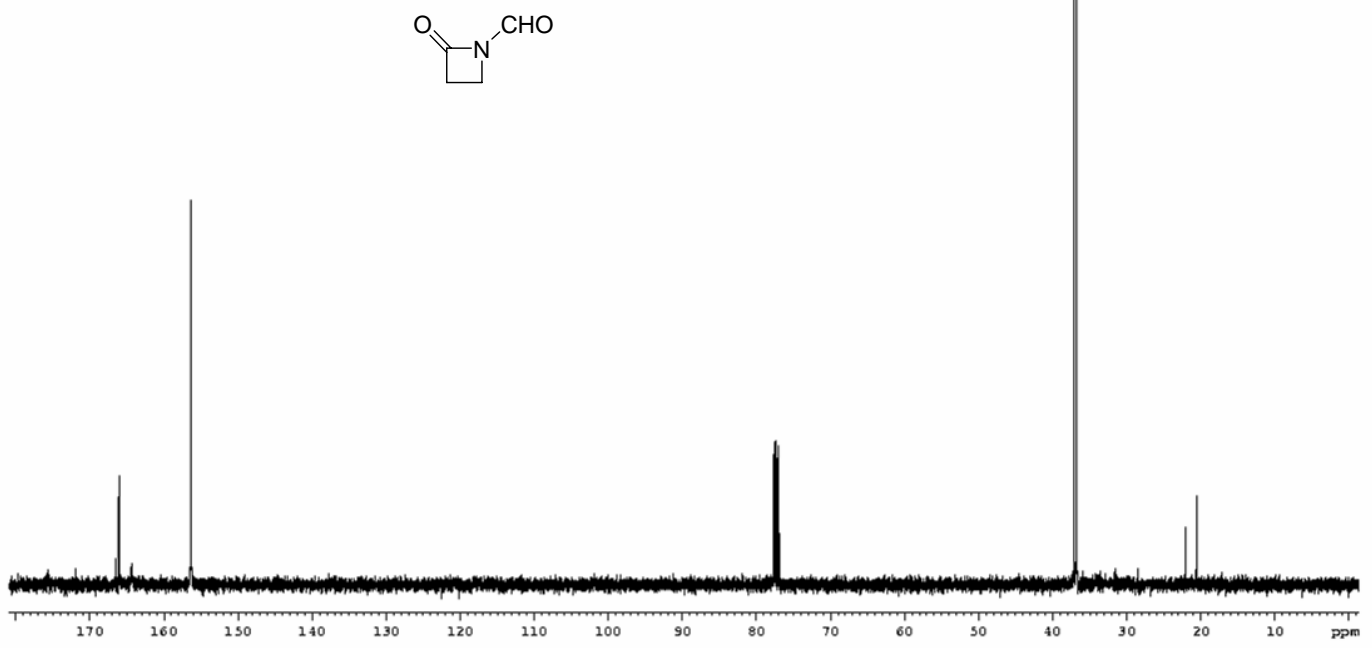
2-Oxo-pyrrolidine-1-carbaldehyde, $9 \mathrm{~b}$.

|

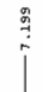

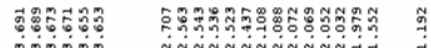

Win

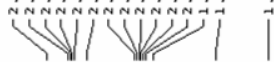

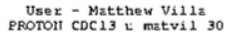

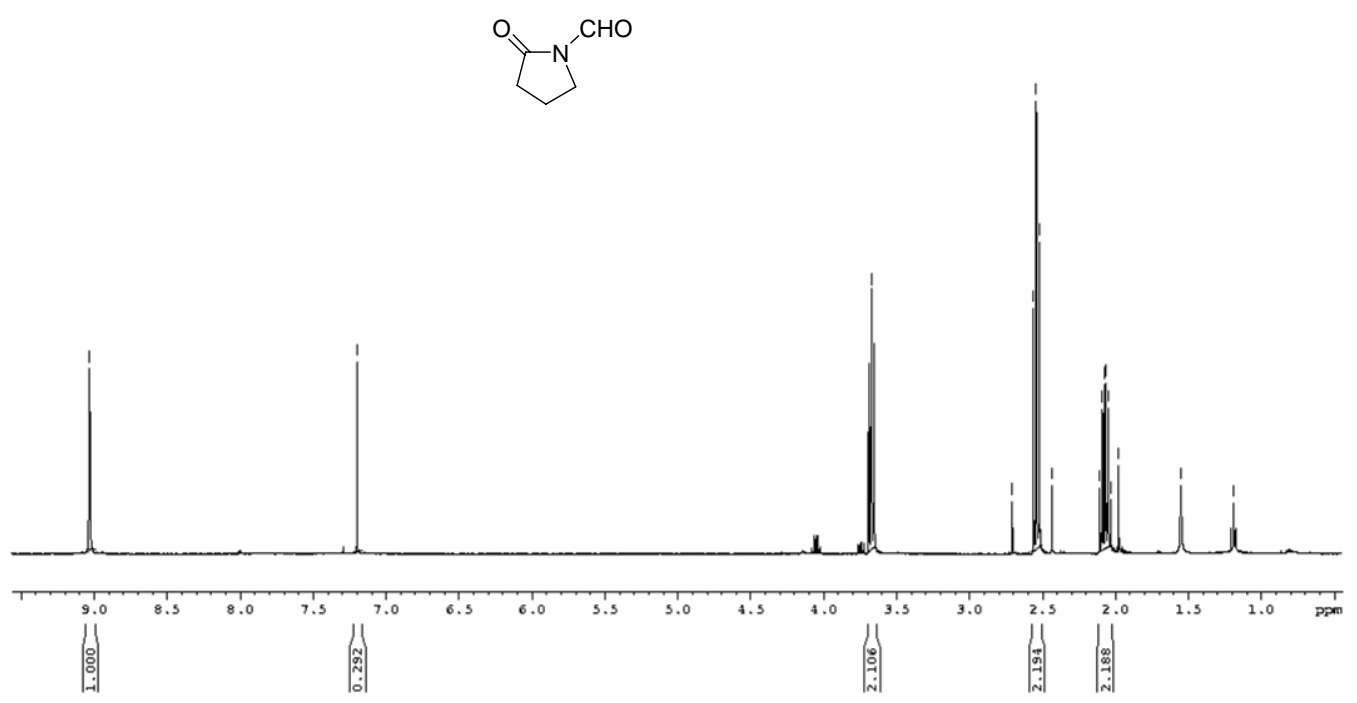

User - Matther Villz
C13CPDA3.q12 CDC13
matvi1 30

$\mid$

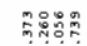

FFF:

V

$\sum^{\mathrm{O}}$

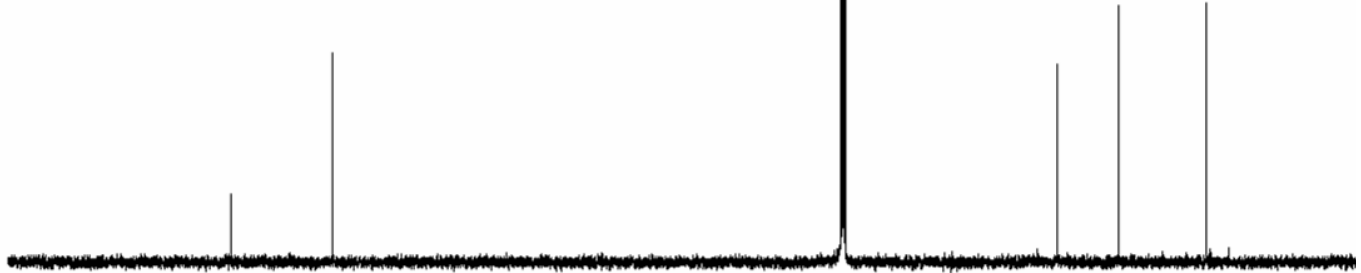

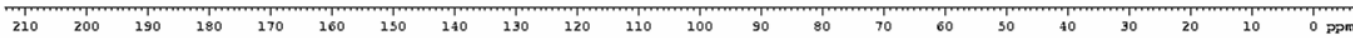


2-Oxo-piperidine-1-carbaldehyde, 9.

Usex - Matther villa
M2-10C
PRoTOI CDC13 $\mathrm{t}$ matvi1

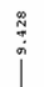

$\left\langle\mathrm{N}^{\mathrm{O}}\right.$
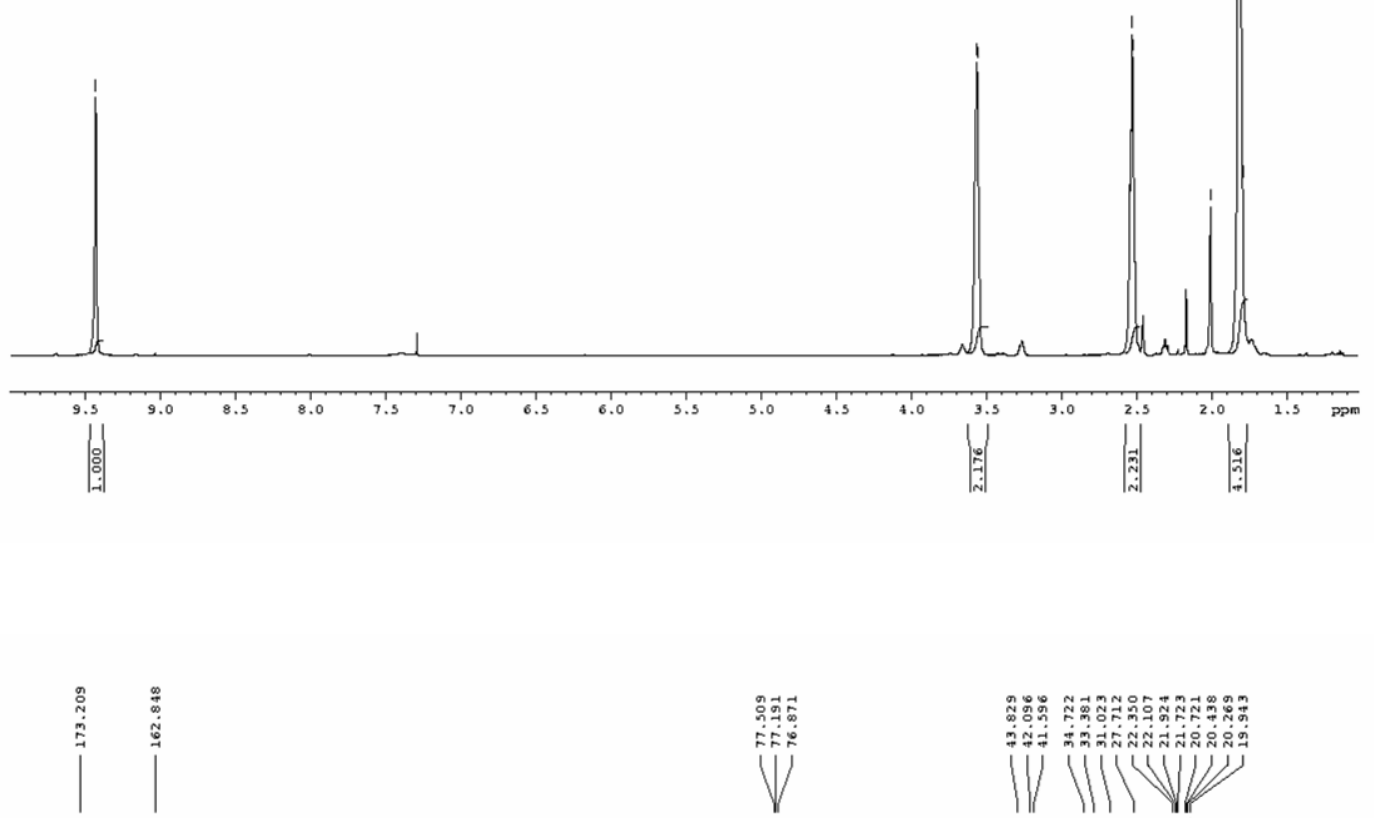

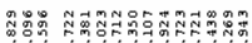

ตูป

IIIUNWW

User - Matther Villa

C13CPD_A3. Q12 MV CDC13 L matvil 26
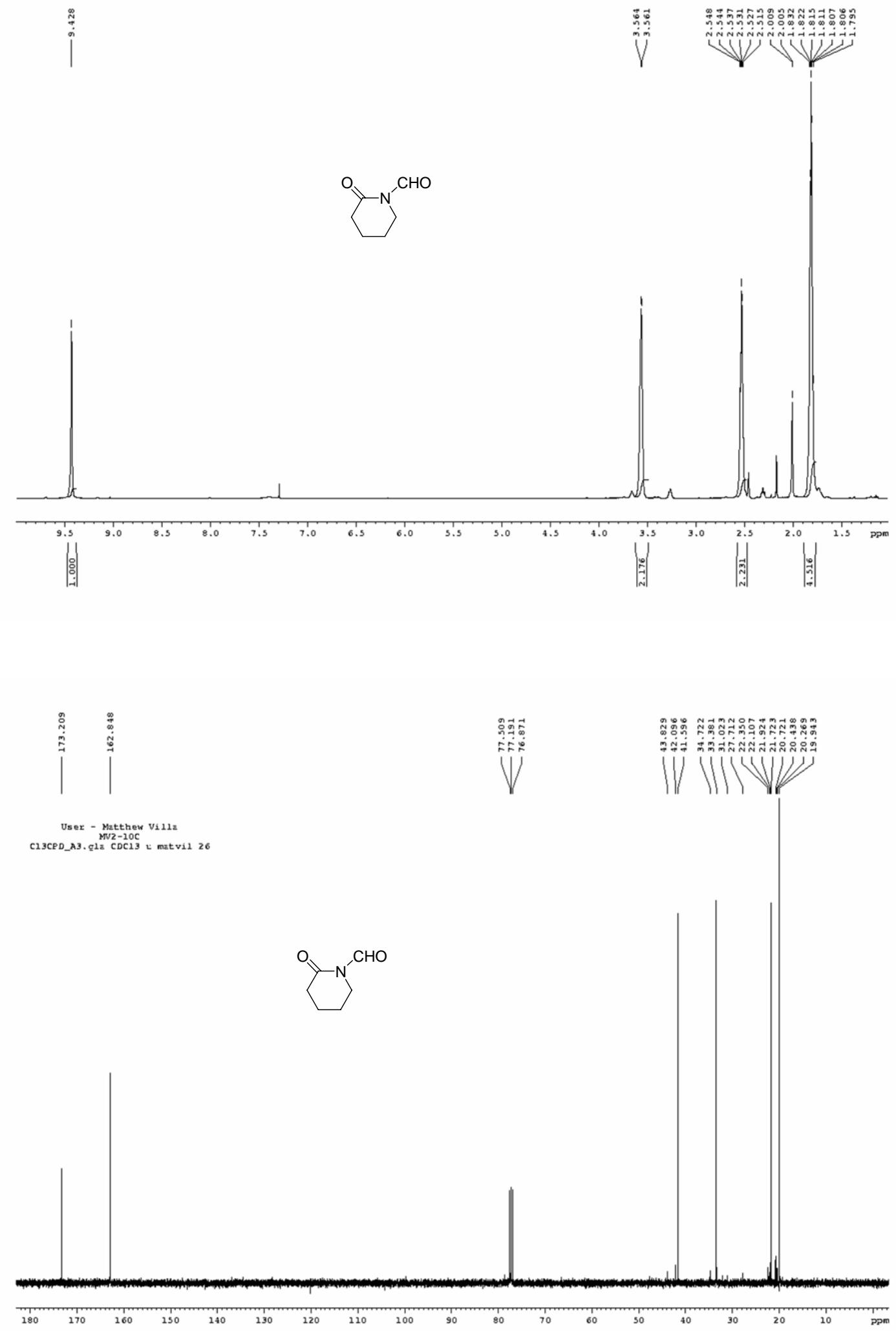

VI 
2-Oxo-azepane-1-carbaldehyde, 9c.

User - Matthew Vil1a

PRoTOI CDC13 2 matvil 4S
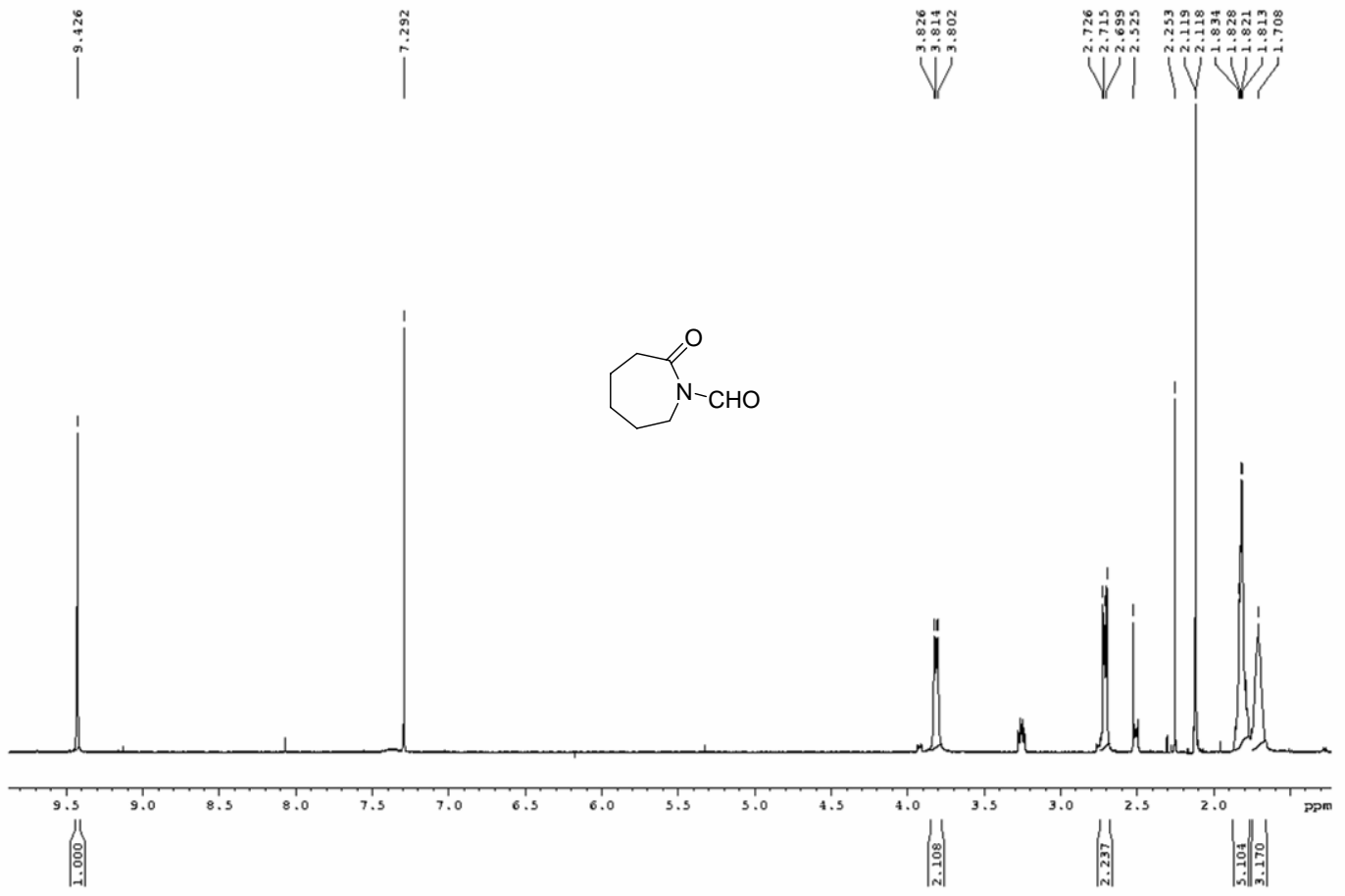

User - Matthew Villa

C13CPD_A3.q1a CDC13 t matvil 42
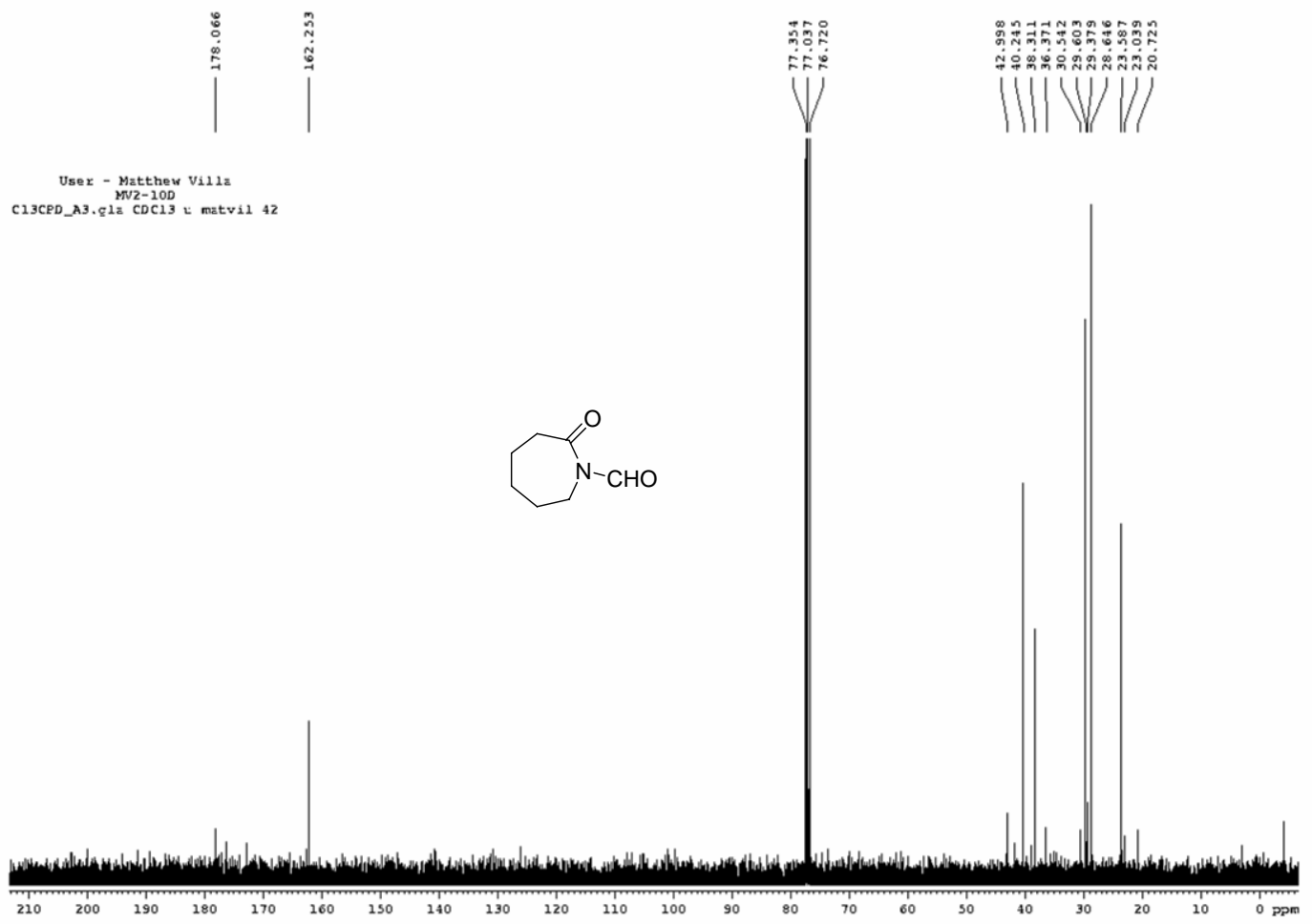
2-Oxo-azocane-1-carbaldehyde, 9d.
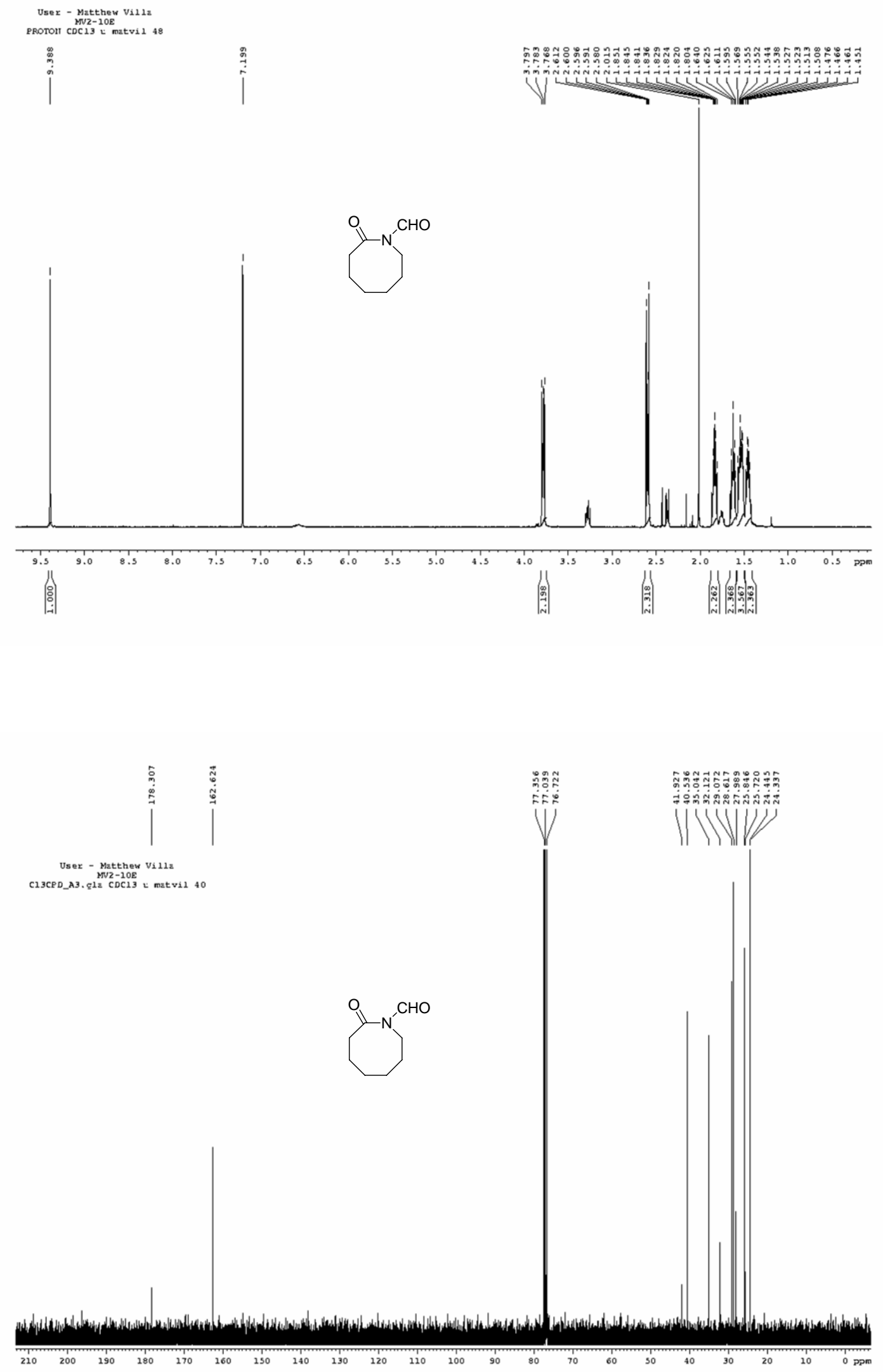
2-Oxo-azonane-1-carbaldehyde, $9 e$.

Usex - Matthew Vill PROTOI CDC13 L matvil SS
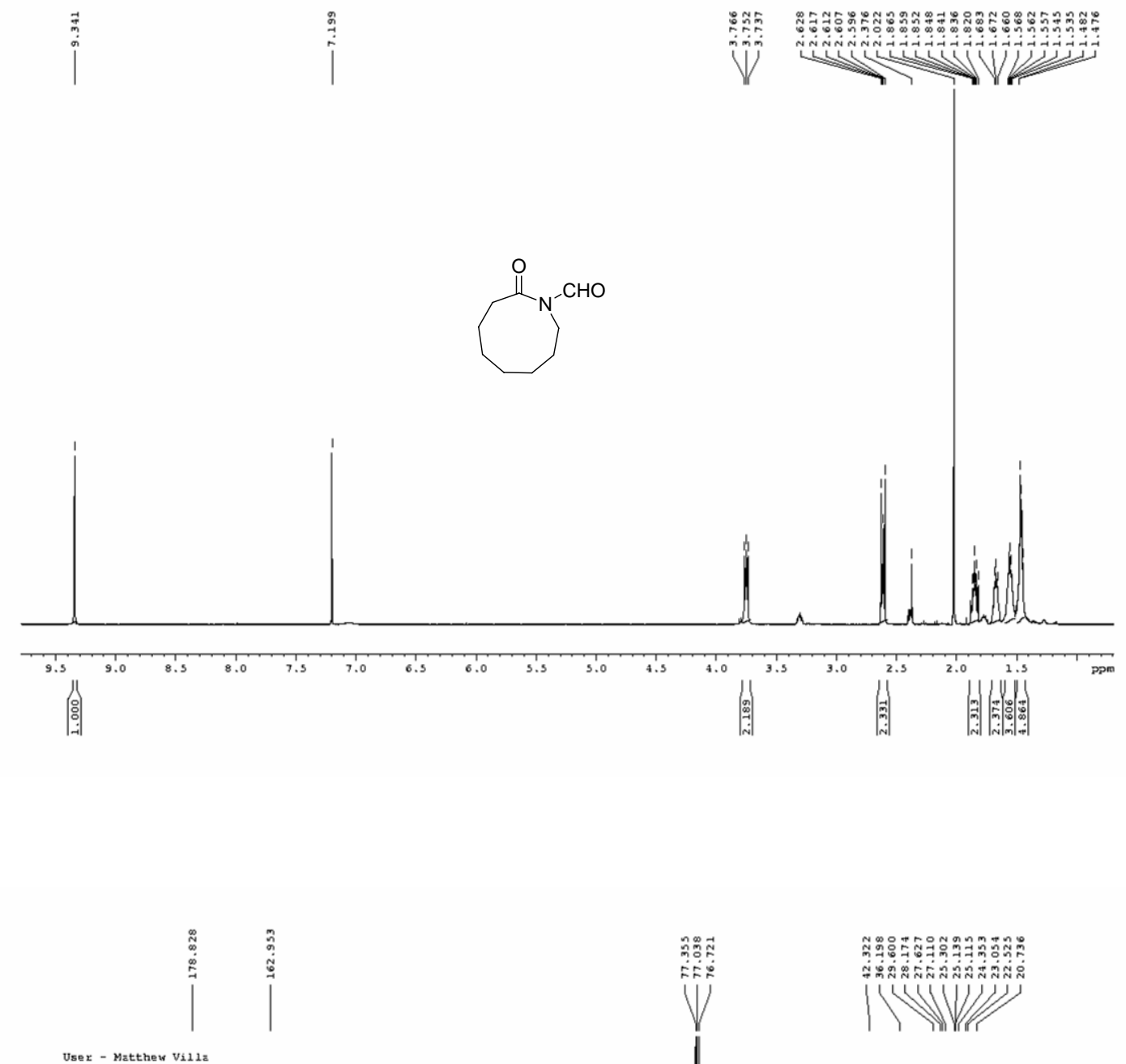

Usex - Matther Villa
MV2-10F
C13CPD_A3.q1a CDC13 L matvil 30

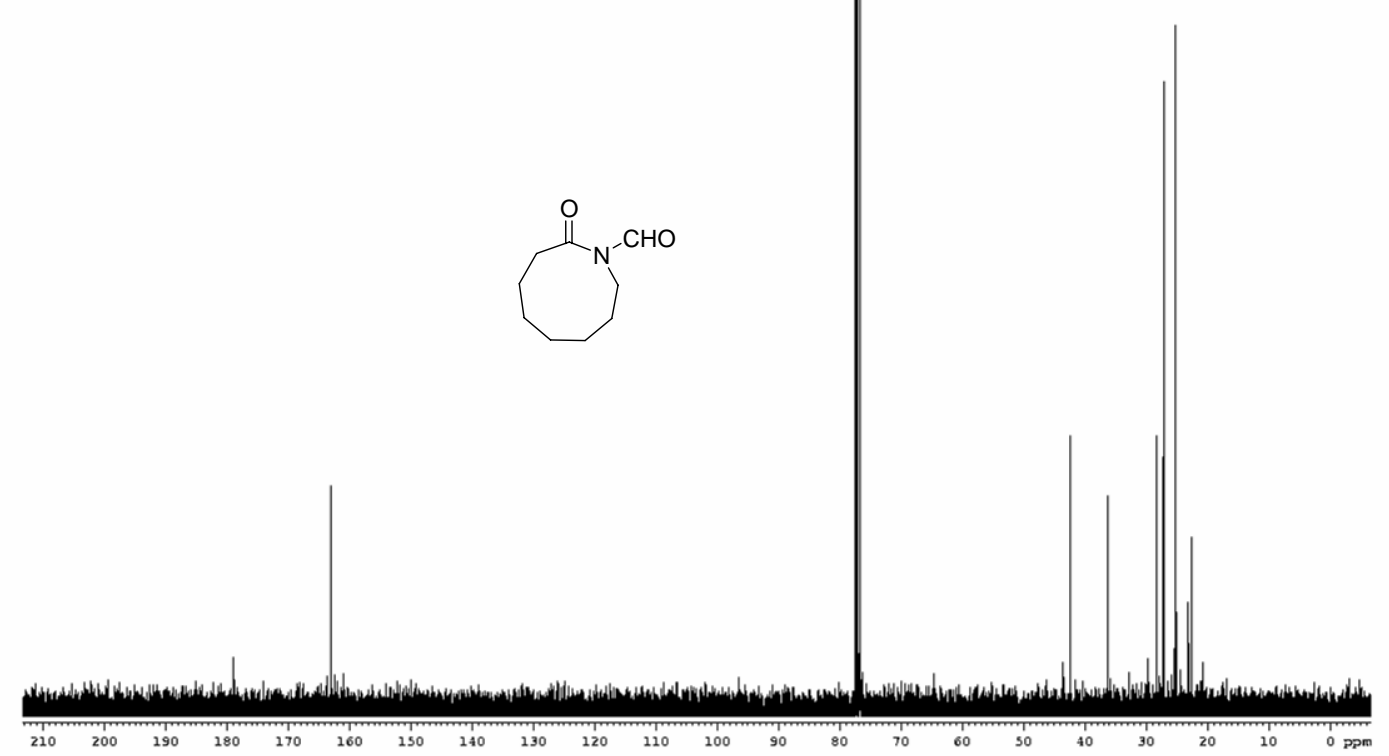


(E)-3-(2-Oxo-azetidin-1-yl)-acrylic acid ethyl ester, 10a.

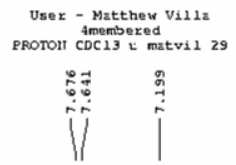
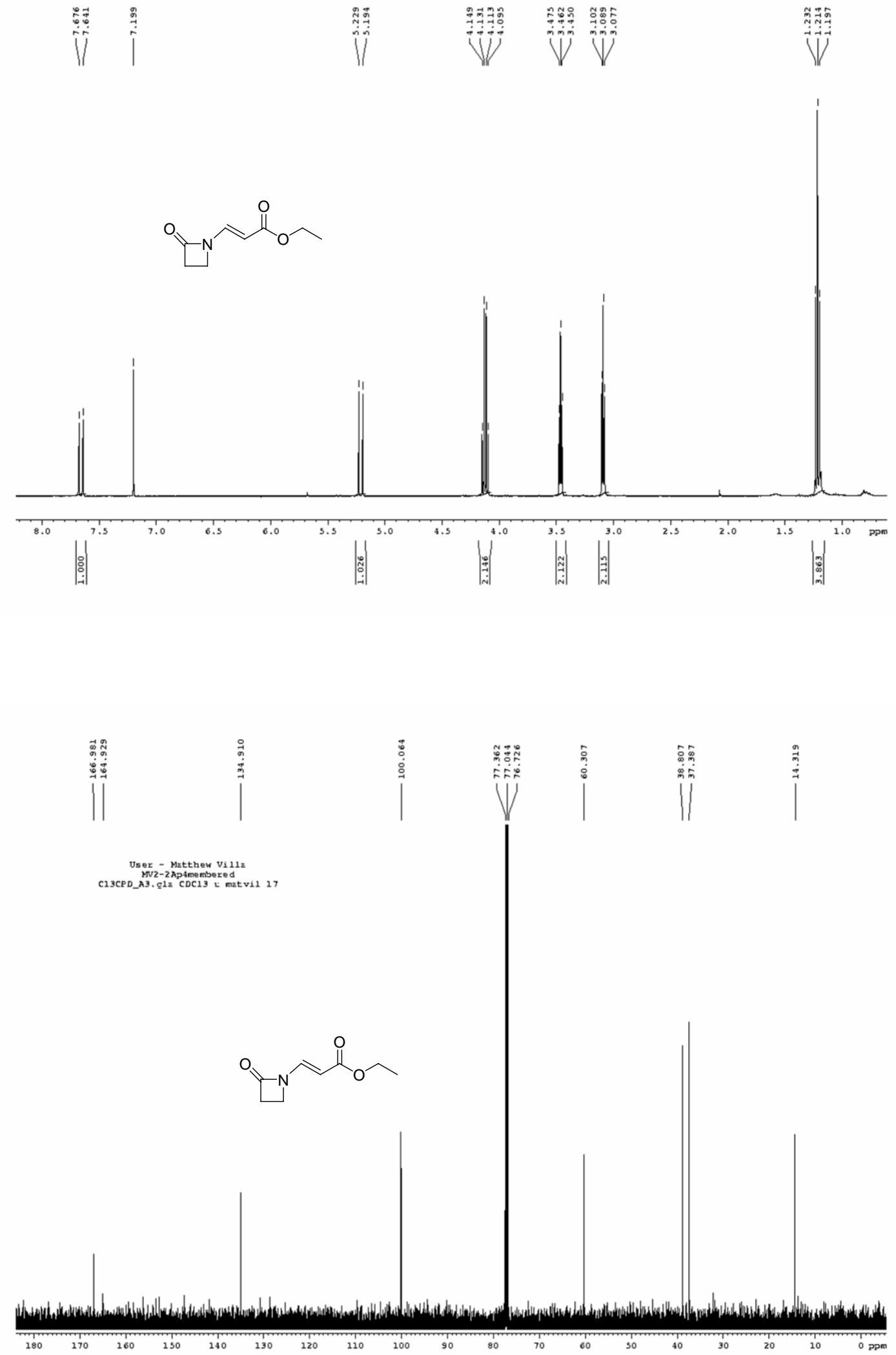
(E)-3-(2-Oxo-pyrrolidin-1-yl)-acrylic acid ethyl ester, $10 \mathrm{~b}$.
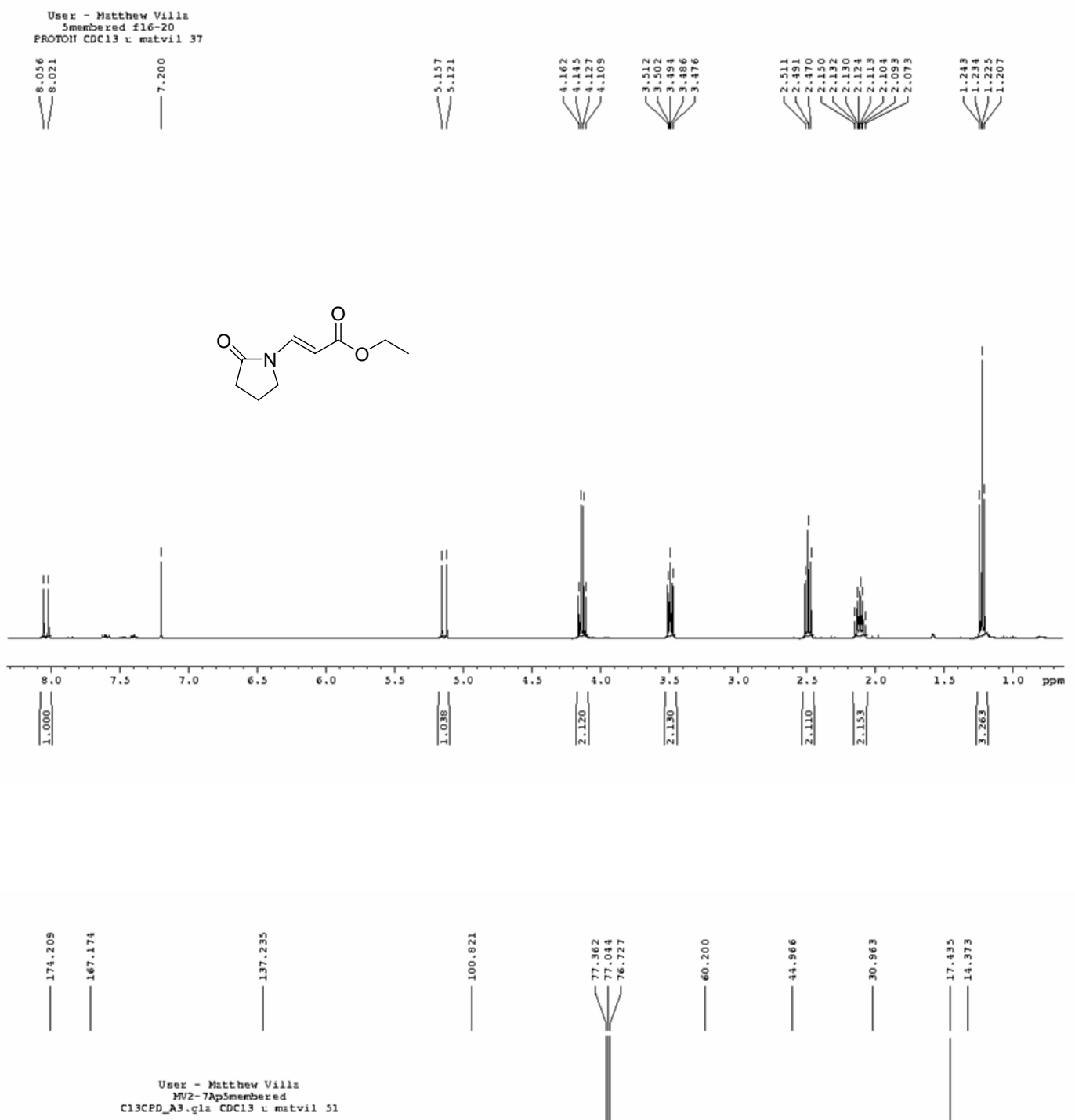

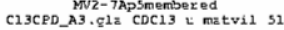

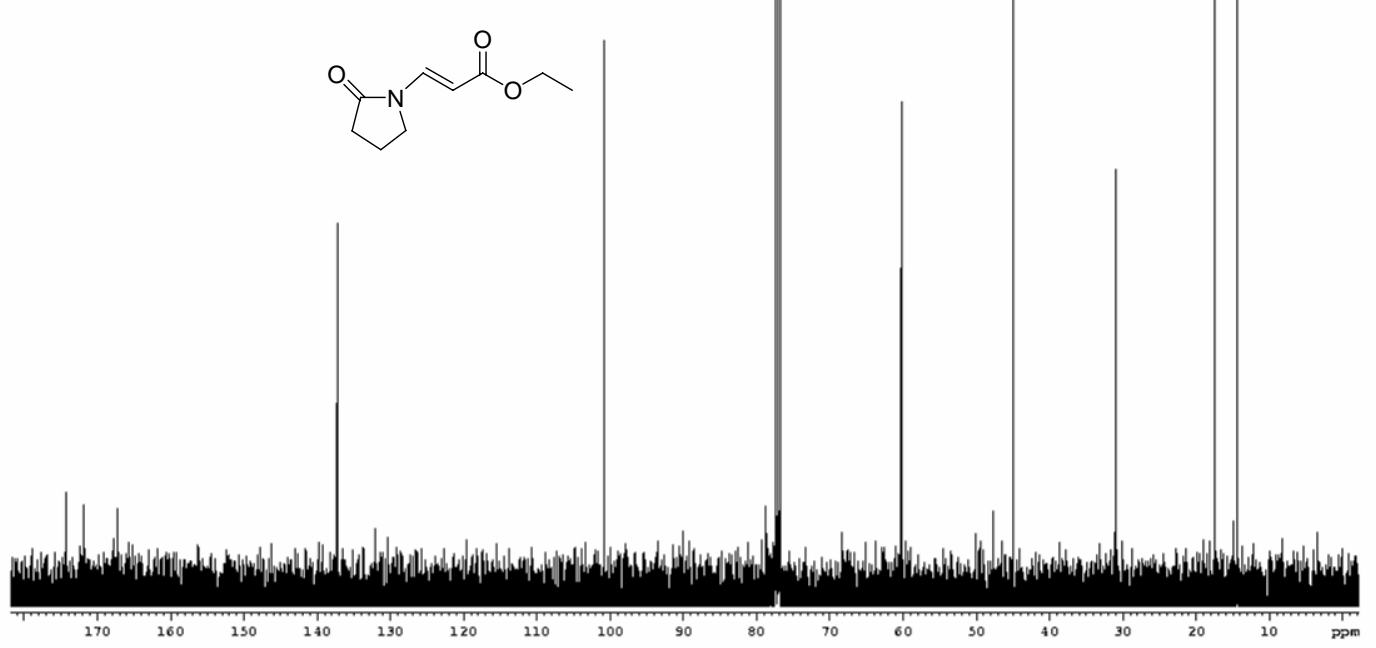


(E)-3-(2-Oxo-piperidin-1-yl)-acrylic acid ethyl ester, 10.
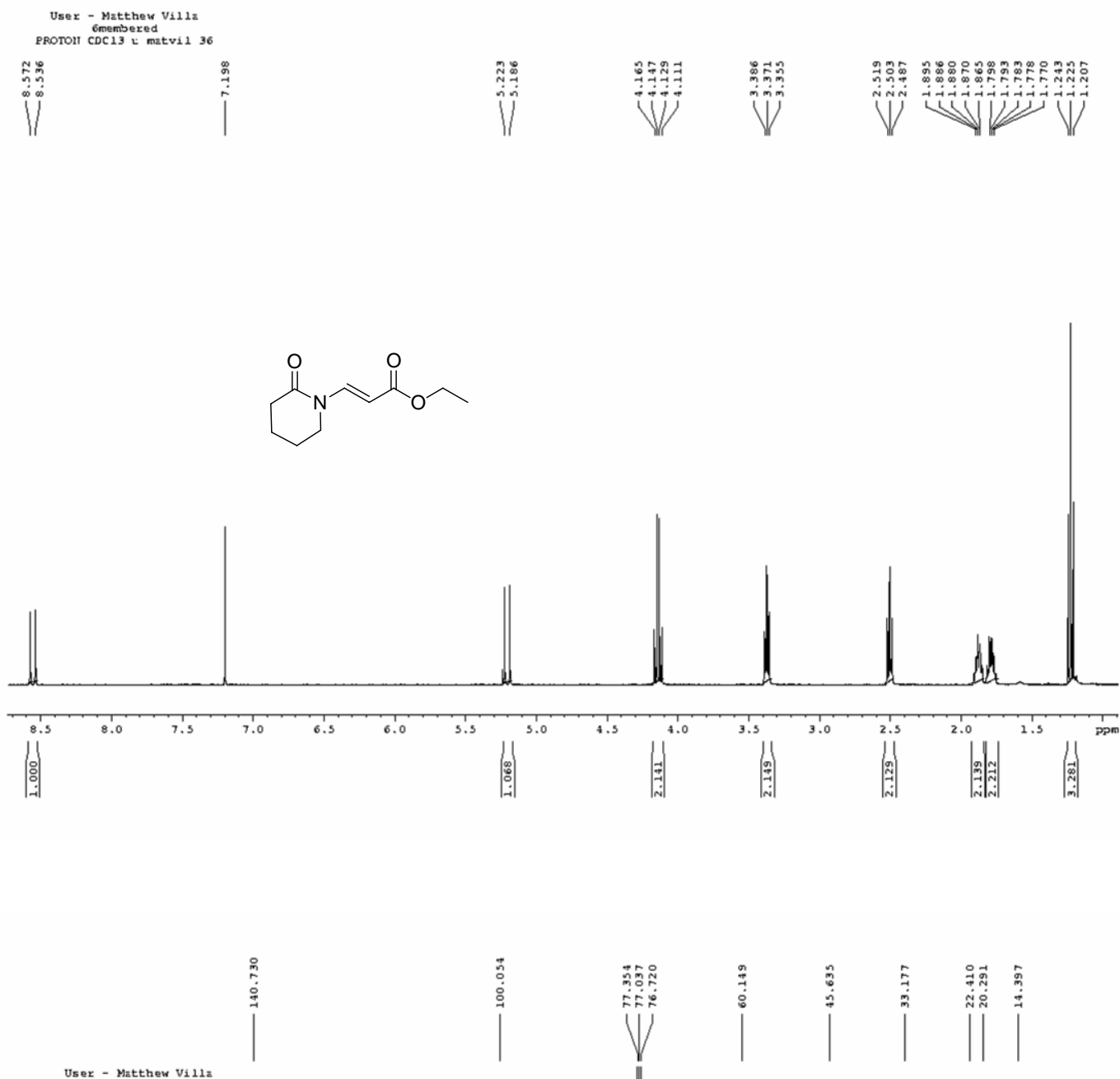

MN179p6membered
C13CPD_A3. G13 CDC13 matyil 24
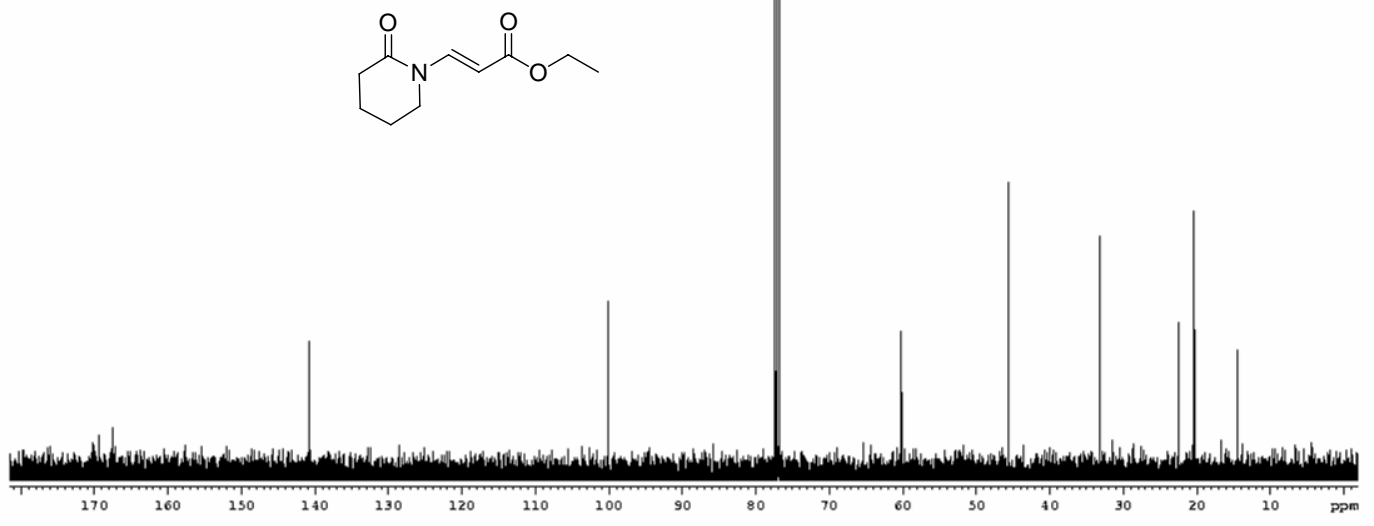
(E)-3-(2-Oxo-azepan-1-yl)-acrylic acid ethyl ester, 10c.

User - Matther Vi11

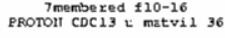

证.

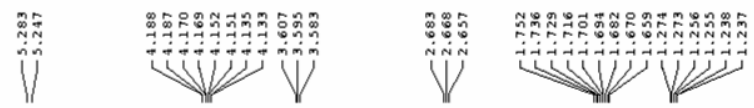

$\left\langle{ }^{N}>\right.$
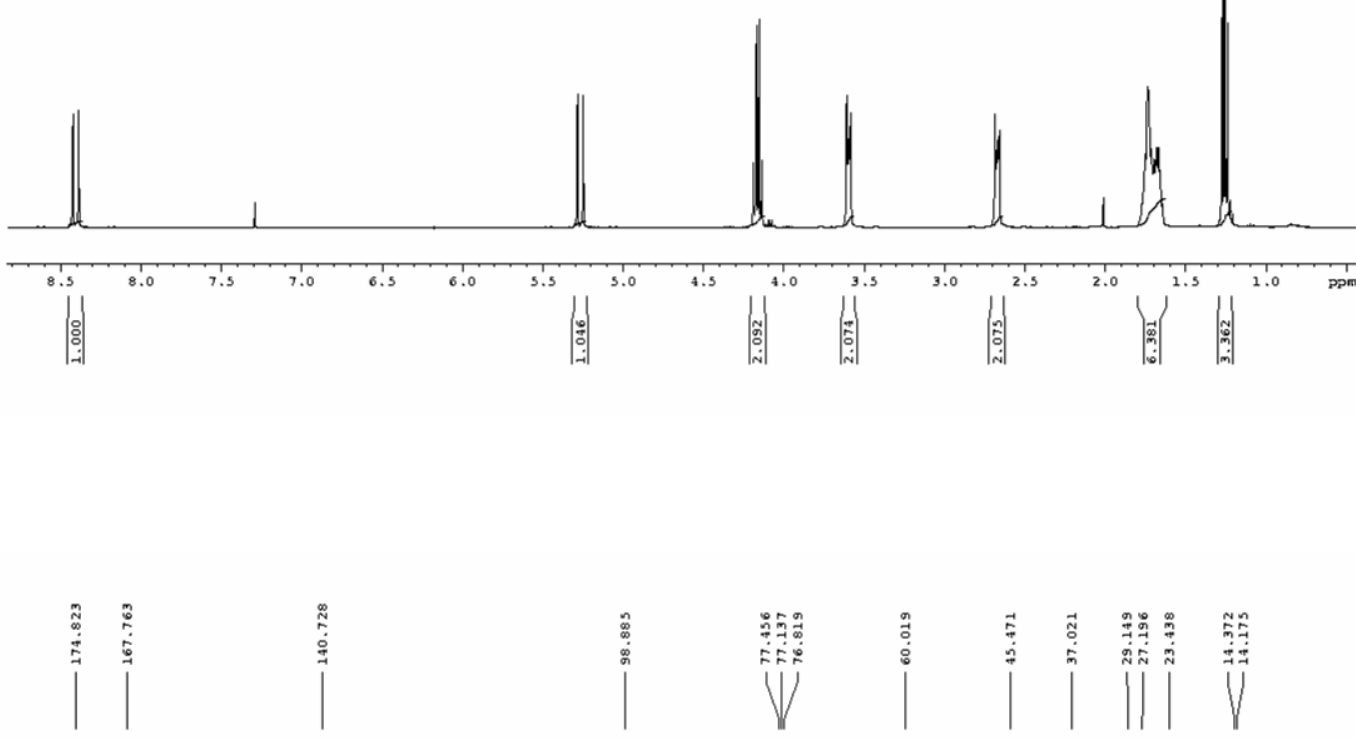

User - Matther Villa C13CPD_A3. Q1ä CDC13 ti matvil 45

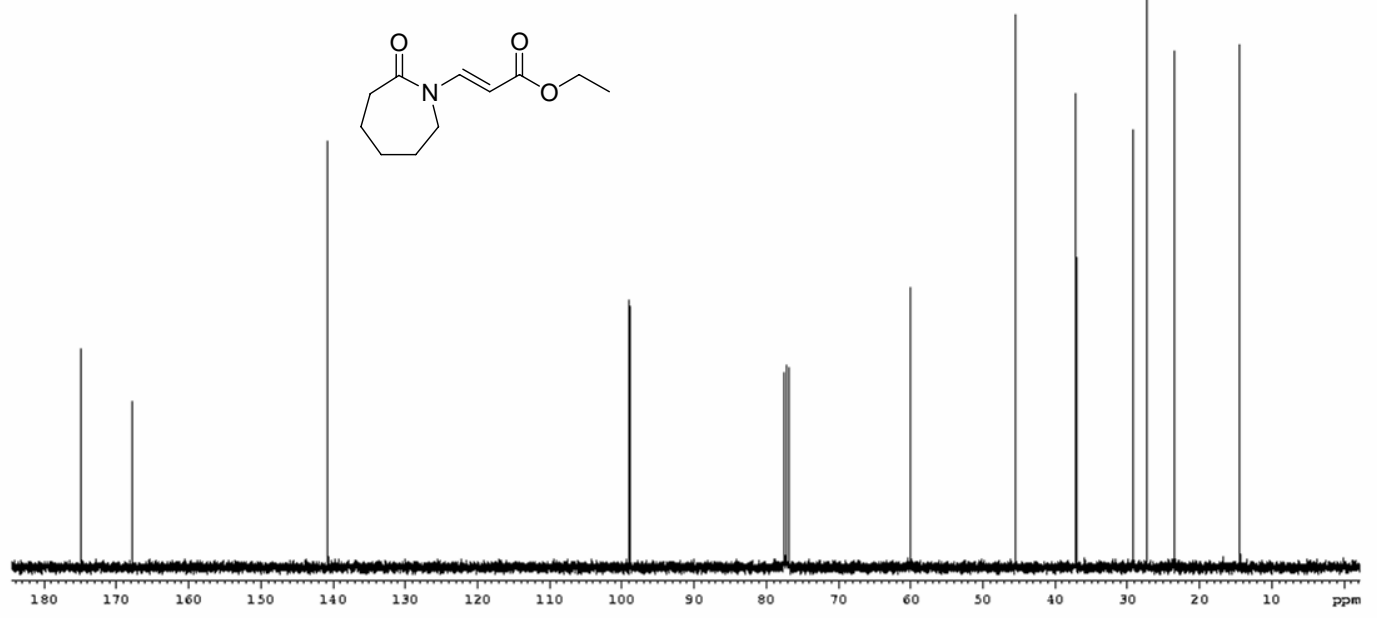


(E)-3-(2-Oxo-azocan-1-yl)-acrylic acid ethyl ester, 10d.
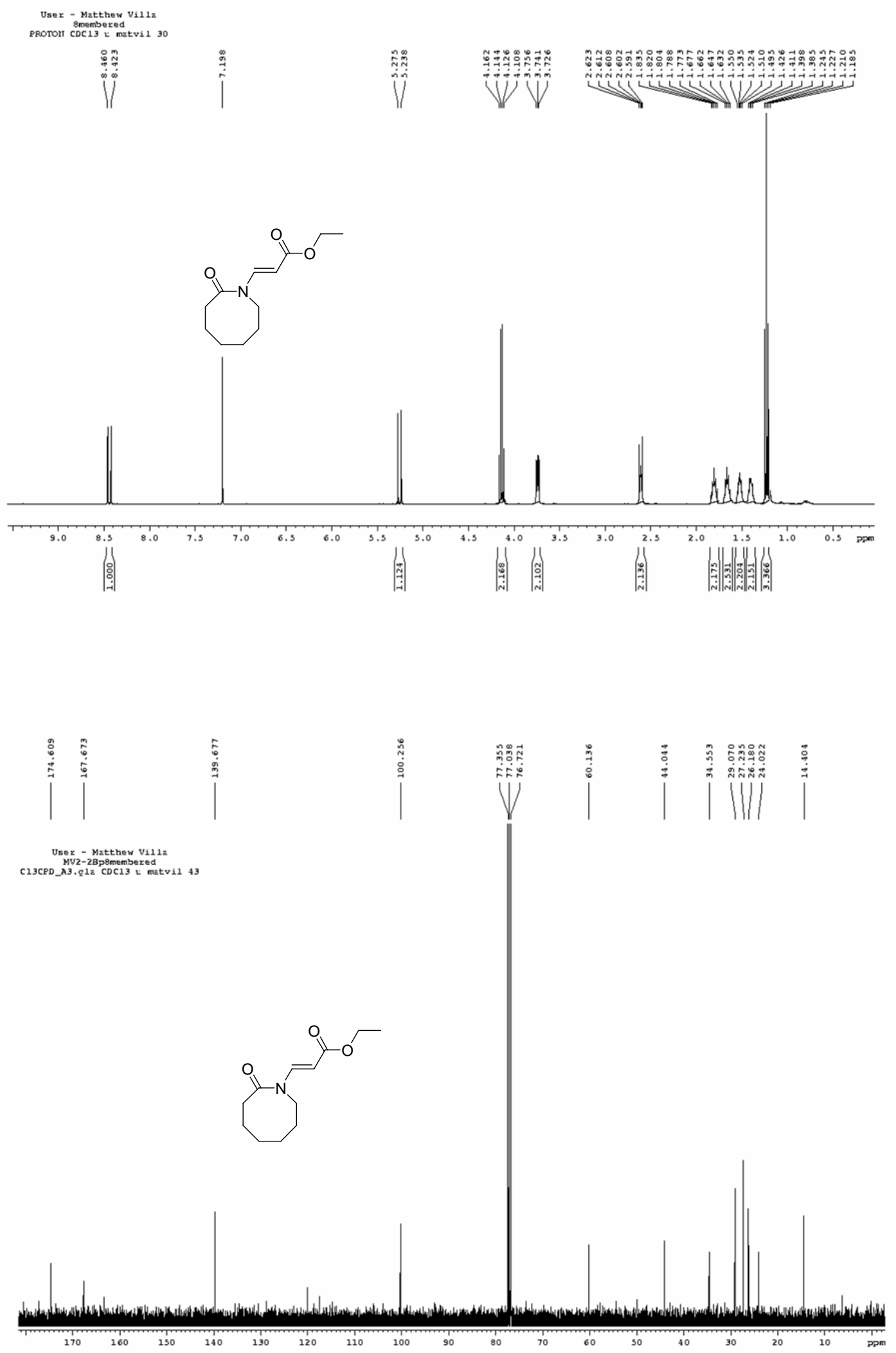
(E)-3-(2-Oxo-azonan-1-yl)-acrylic acid ethyl ester, 10e.

User - Matthew Villa

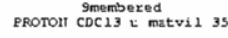

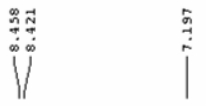

ฟิ่
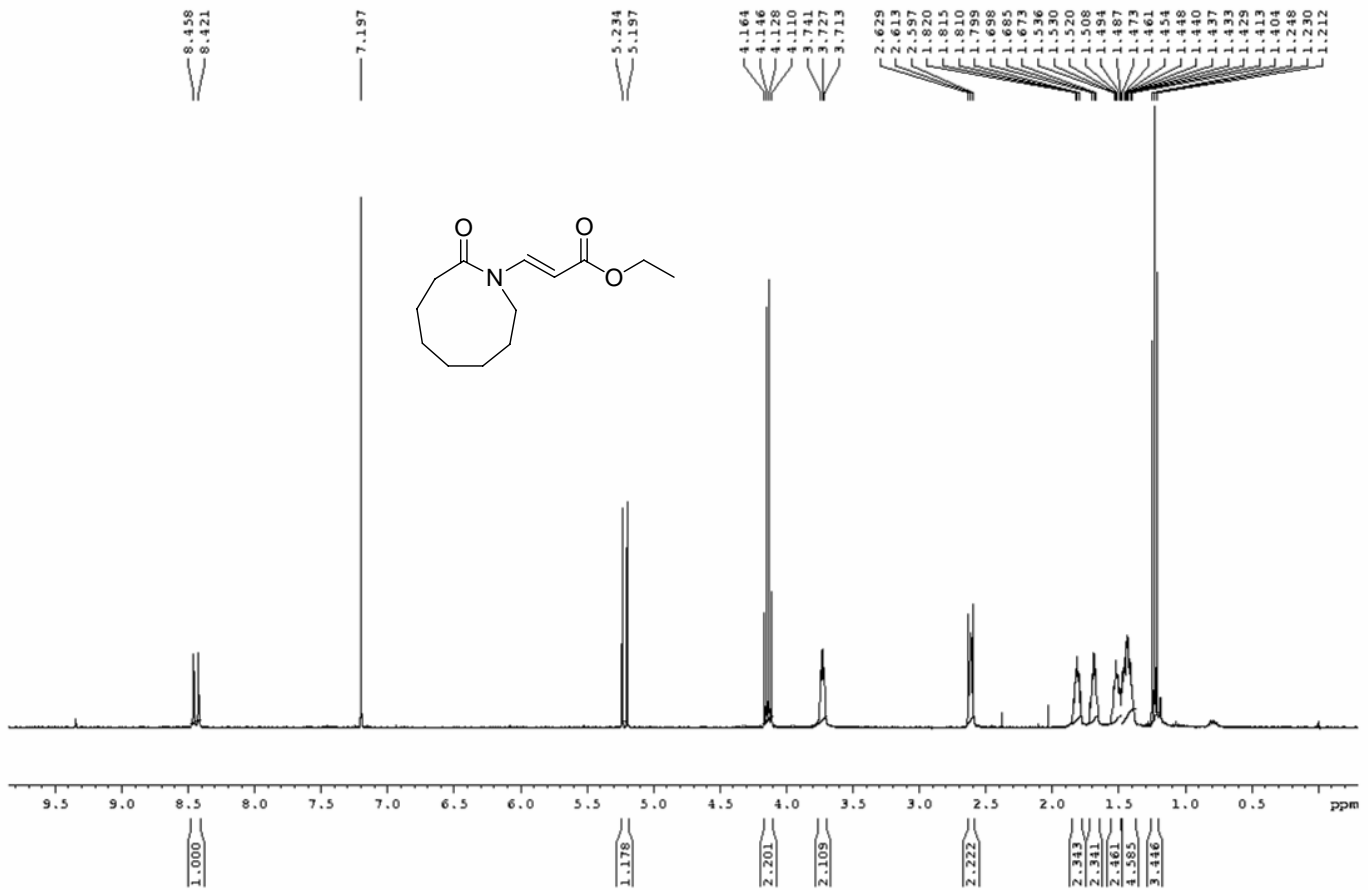

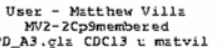

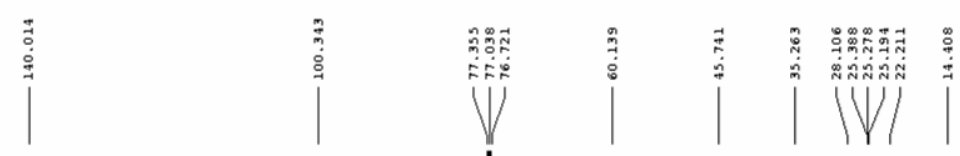

C13CPD_A3.g1a CDC13 ti matvil 36<smiles>CCOC(=O)/C=C/N1CCCCCCCC1=O</smiles>

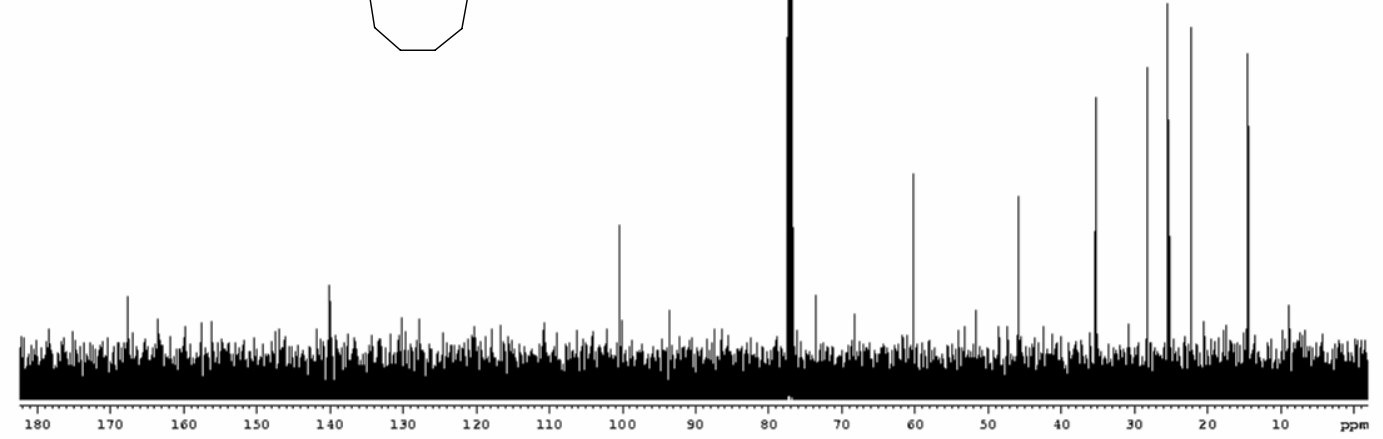


$N$-Formylbenzamide, 12a.

User - Matther Vil1a
MN2-79-12ps3
PROTOH CDC13 matvi1

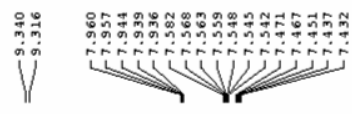

Vij

|<smiles>O=CNC(=O)c1ccccc1</smiles>
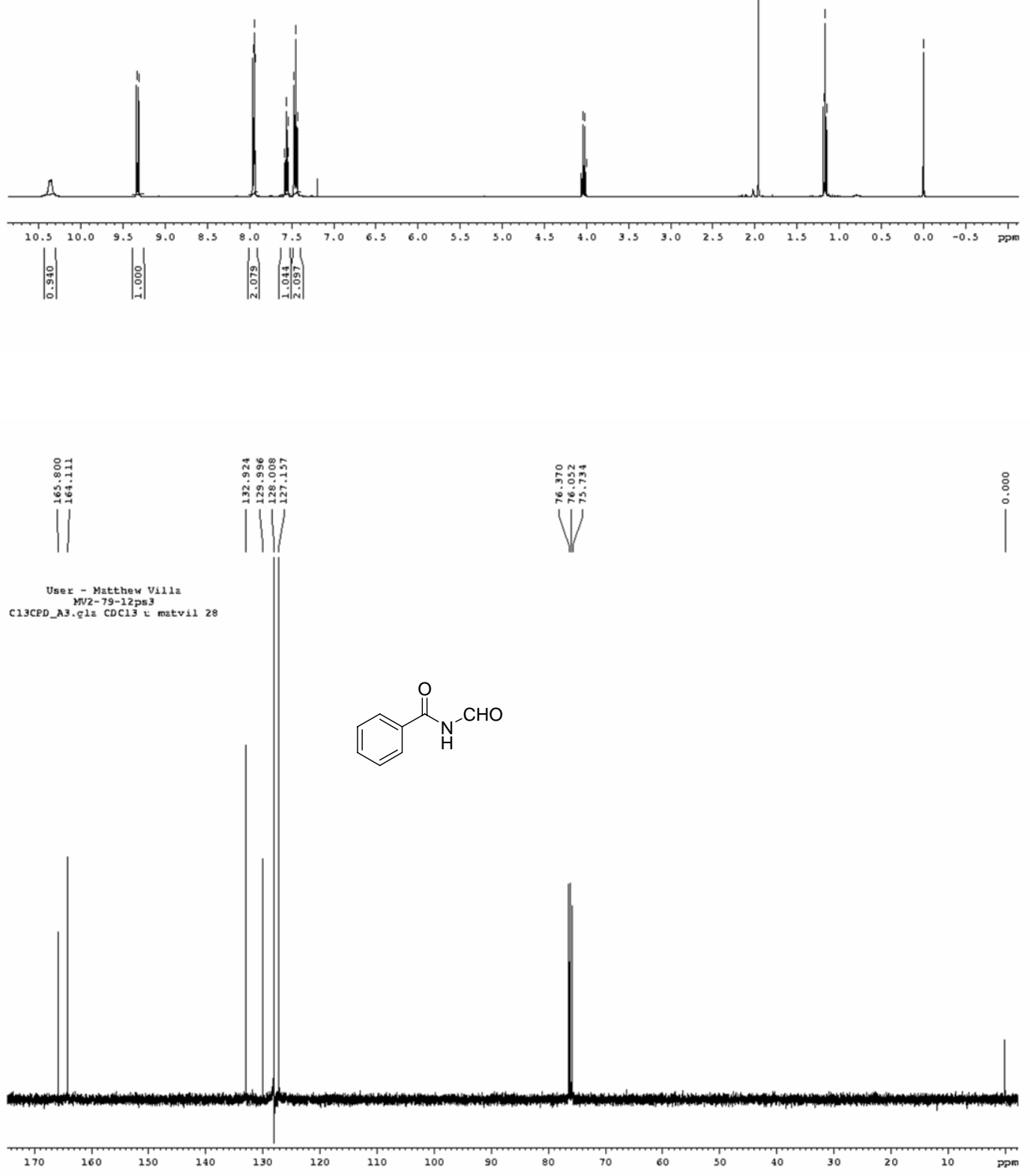
(Z)-3-Benzoylamino-acrylic acid ethyl ester, 13a (cis).
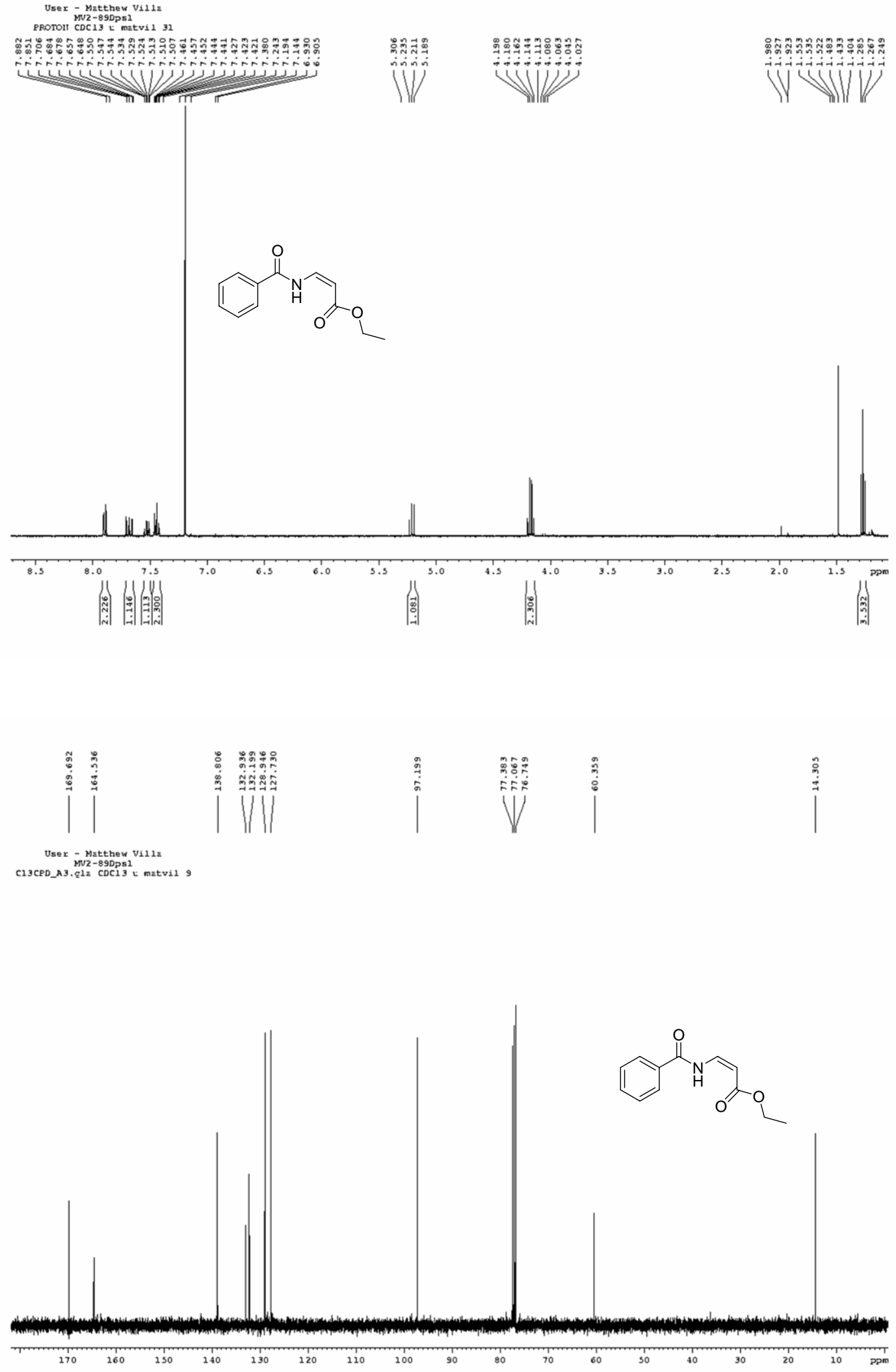

20 
(E)-3-Benzoylamino-acrylic acid ethyl ester, 13a (trans).
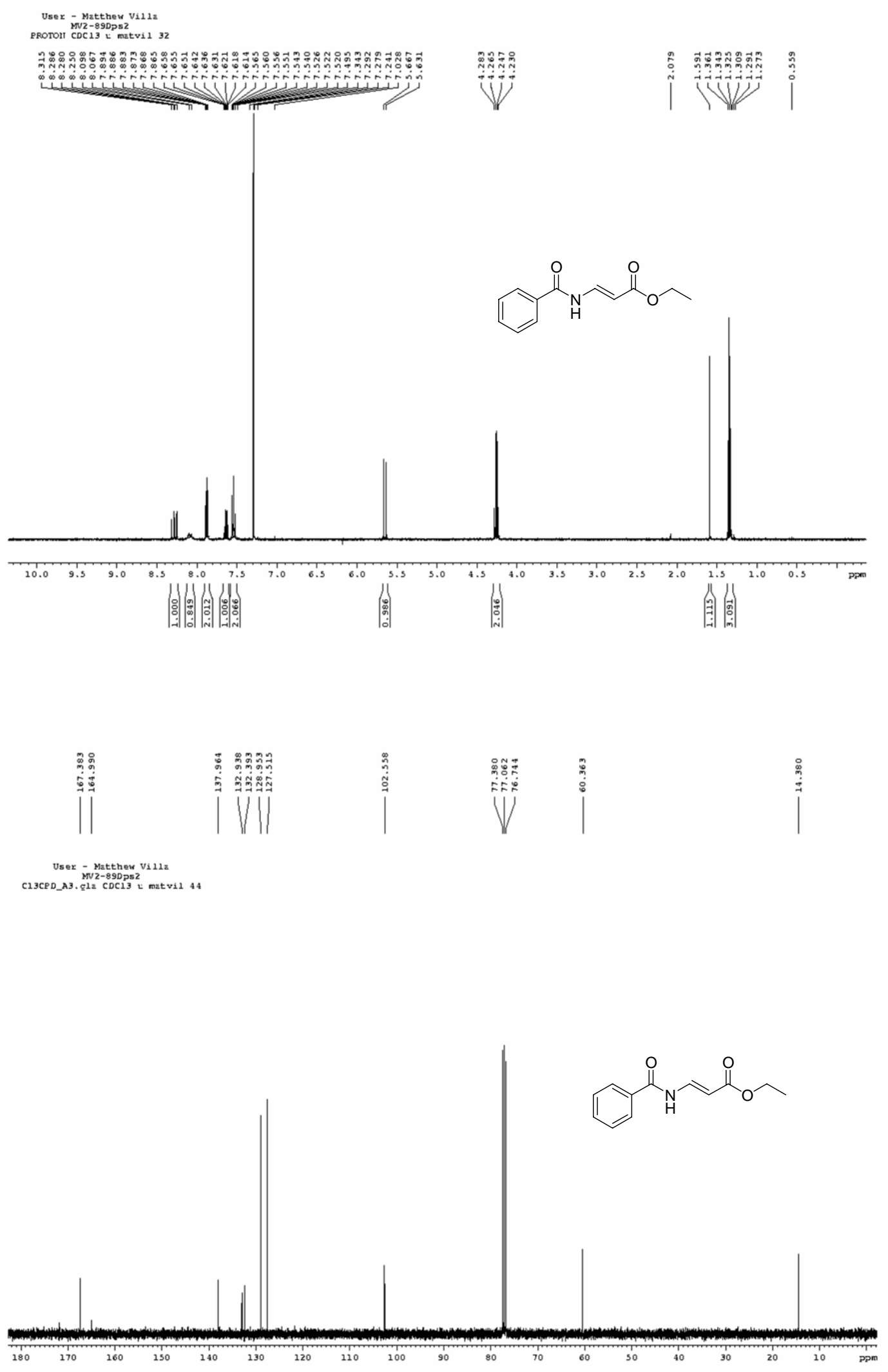
(Z)-3-Benzoylamino-2-methyl-acrylic acid ethyl ester, 14a (cis).

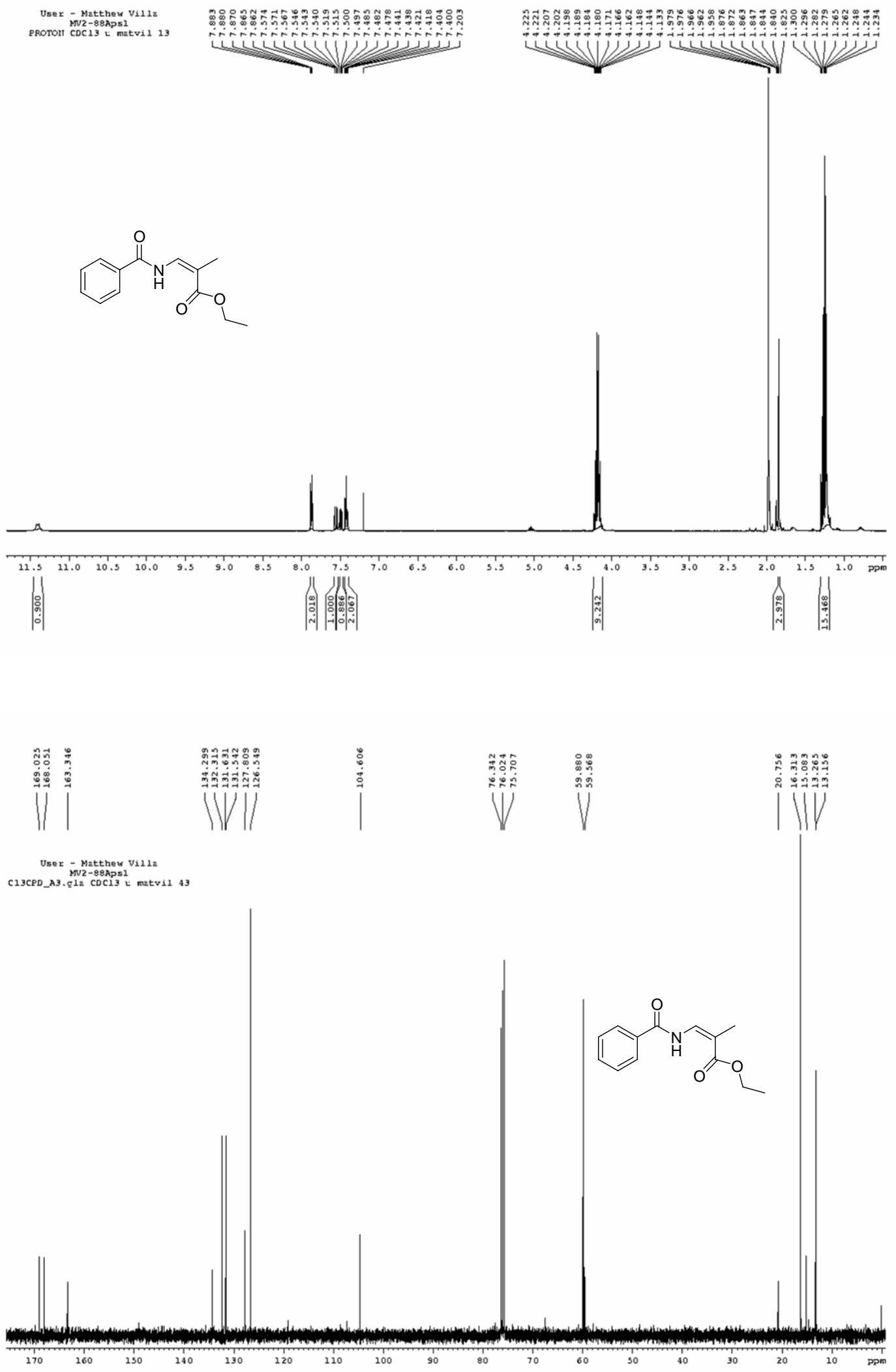


(E)-3-Benzoylamino-2-methyl-acrylic acid ethyl ester, 14a (trans).

Usex - Matthew Vilia

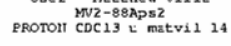
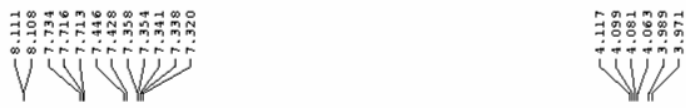

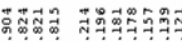
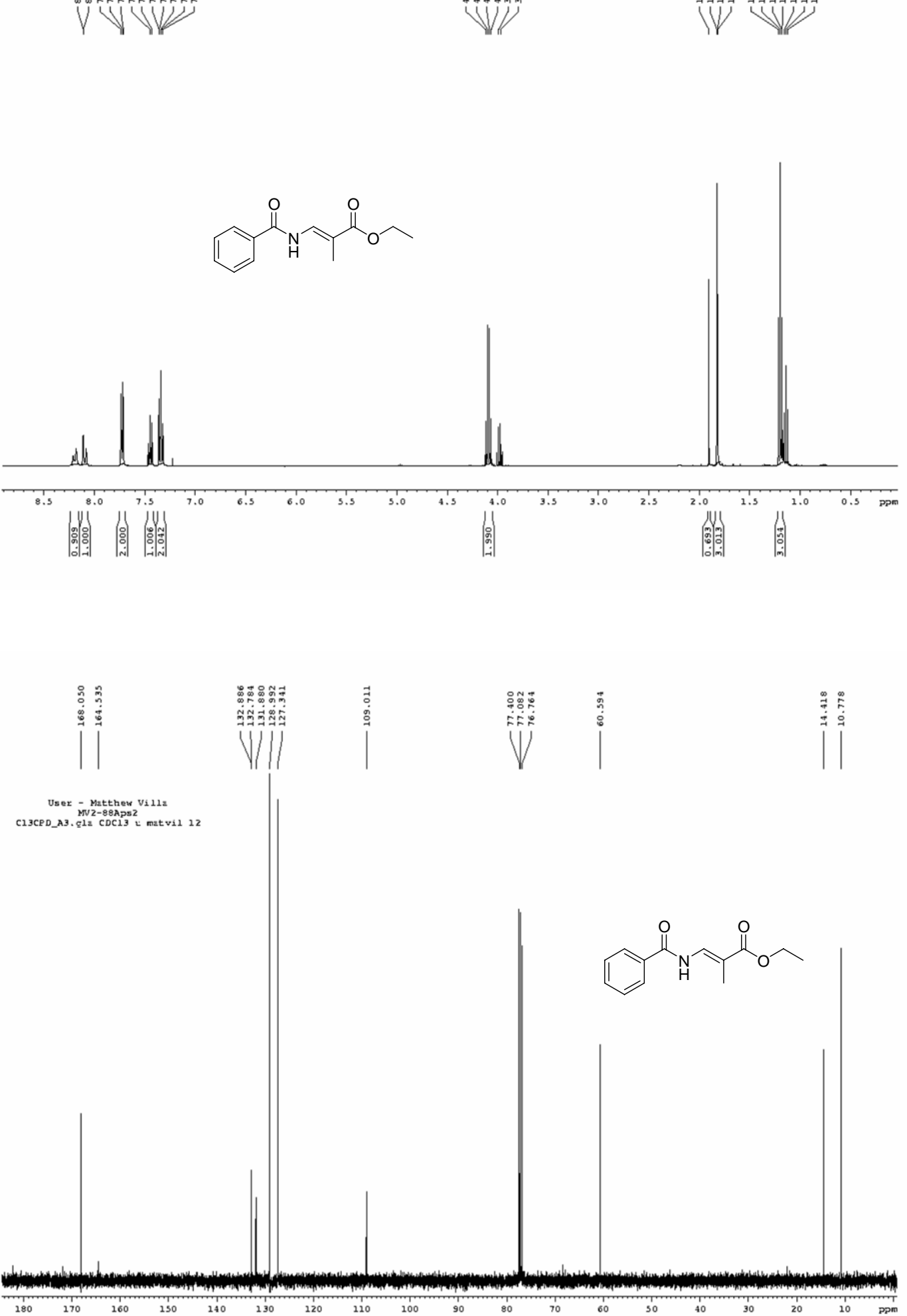
$N$-Formylnicotinamide, $12 b$.
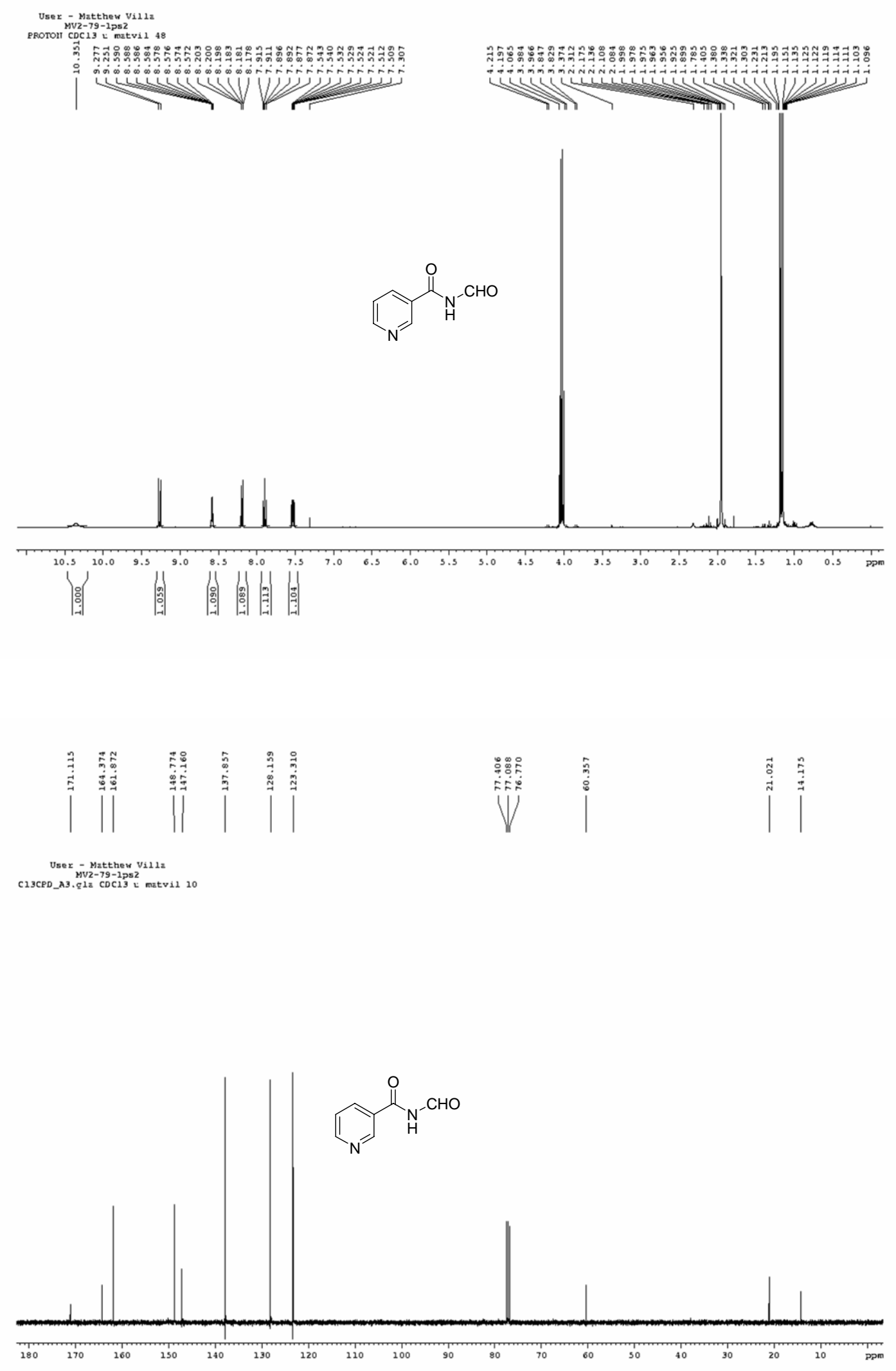
(Z)-3-[(Pyridine-3-carbonyl)-amino]-acrylic acid ethyl ester, 13b (cis).

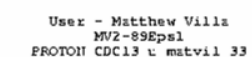

:

$\longrightarrow$ W N
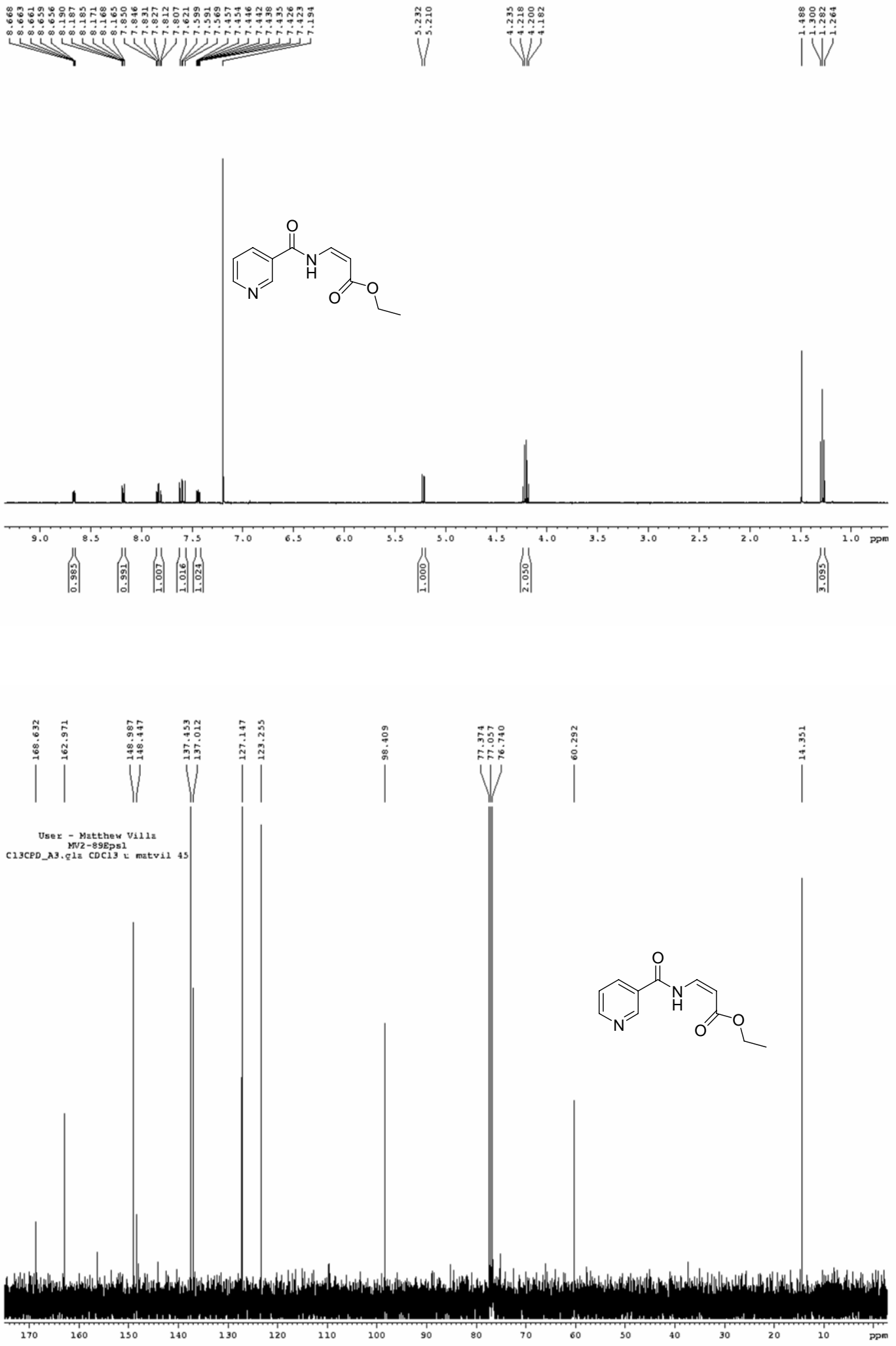
(E)-3-[(Pyridine-3-carbonyl)-amino]-acrylic acid ethyl ester, 13b (trans).

User - Matther Vi11a PROTOII CDC13 L matvi1 34

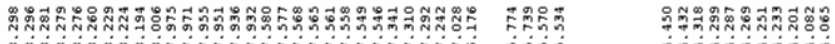

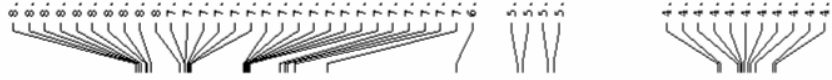

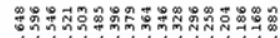

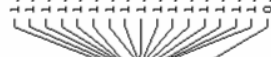

$\ldots$ Wil
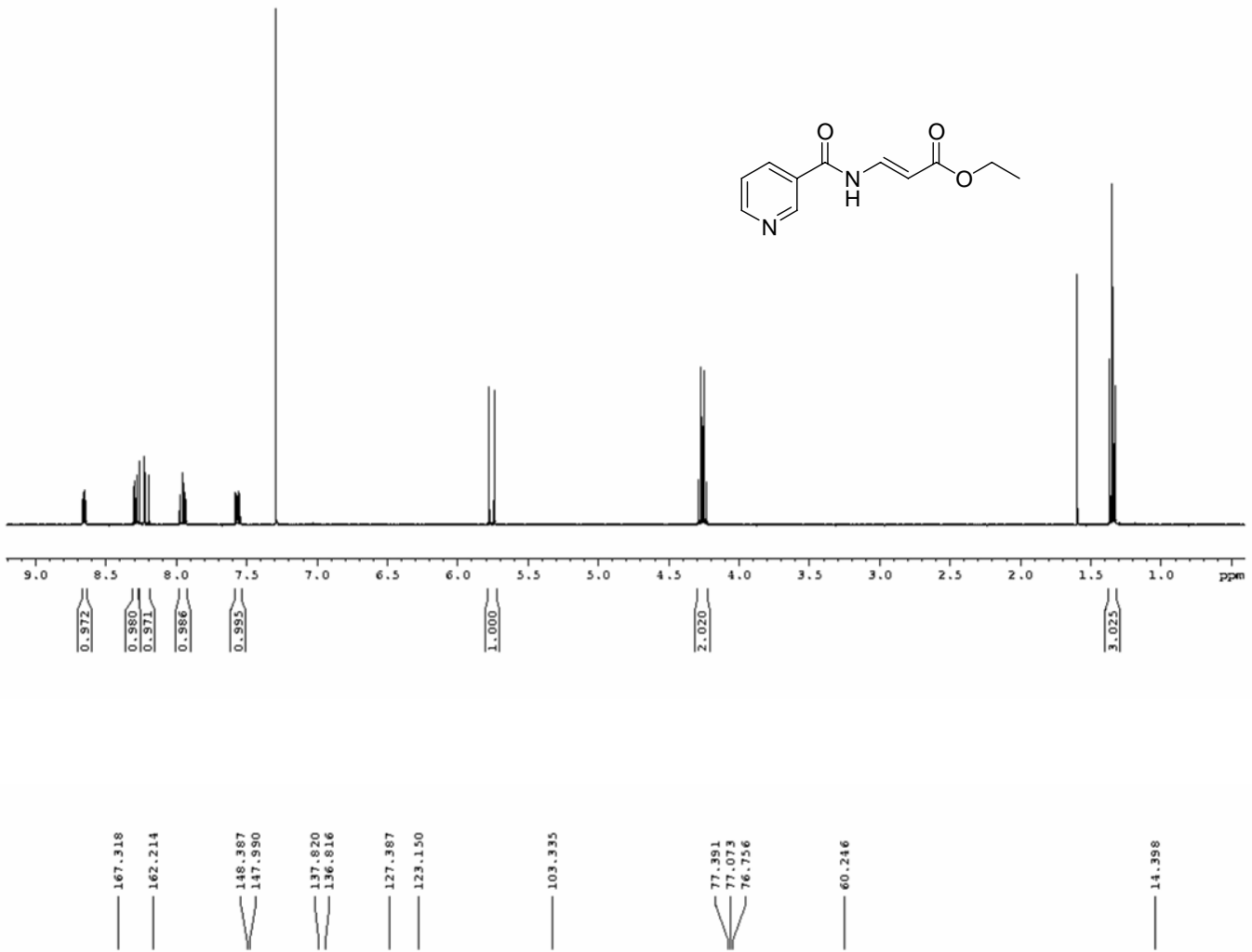

Vin

$\stackrel{\infty}{\stackrel{\infty}{2}}$

C13CPD_A3. Q1a CDC13 2 matvi1 10

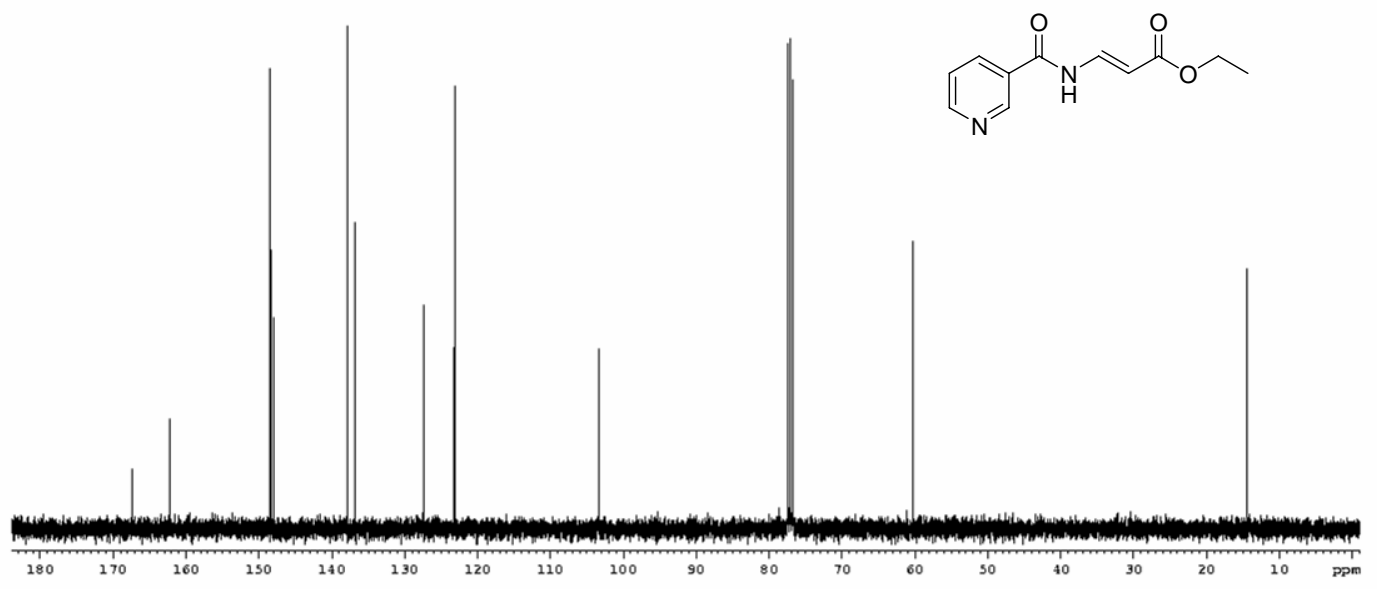


(Z)-2-Methyl-3-[(pyridine-3-carbonyl)-amino]-acrylic acid ethyl ester, 14b (cis).
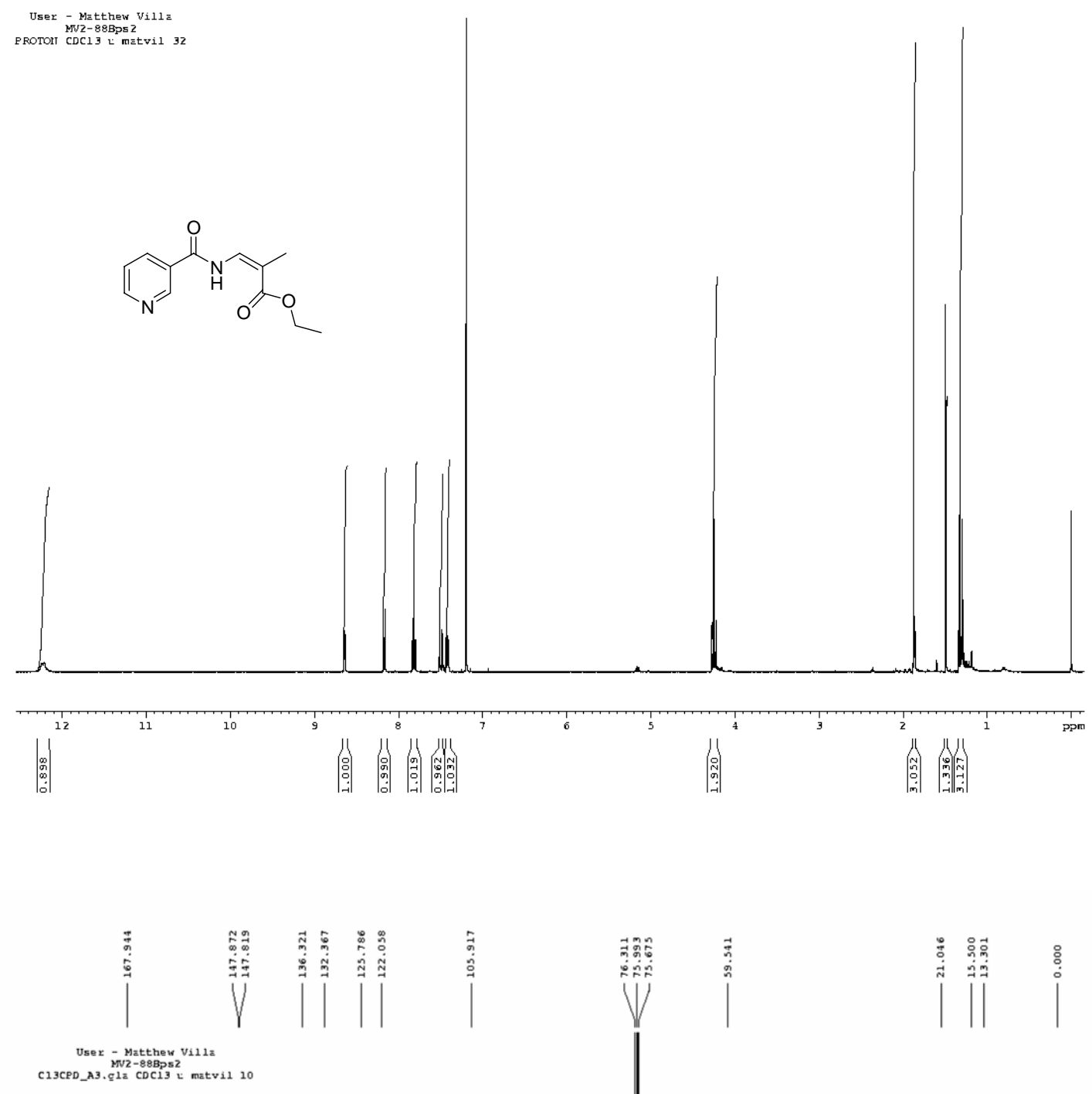
C13CPD_A3. Q1a CDC13 L matvil 10

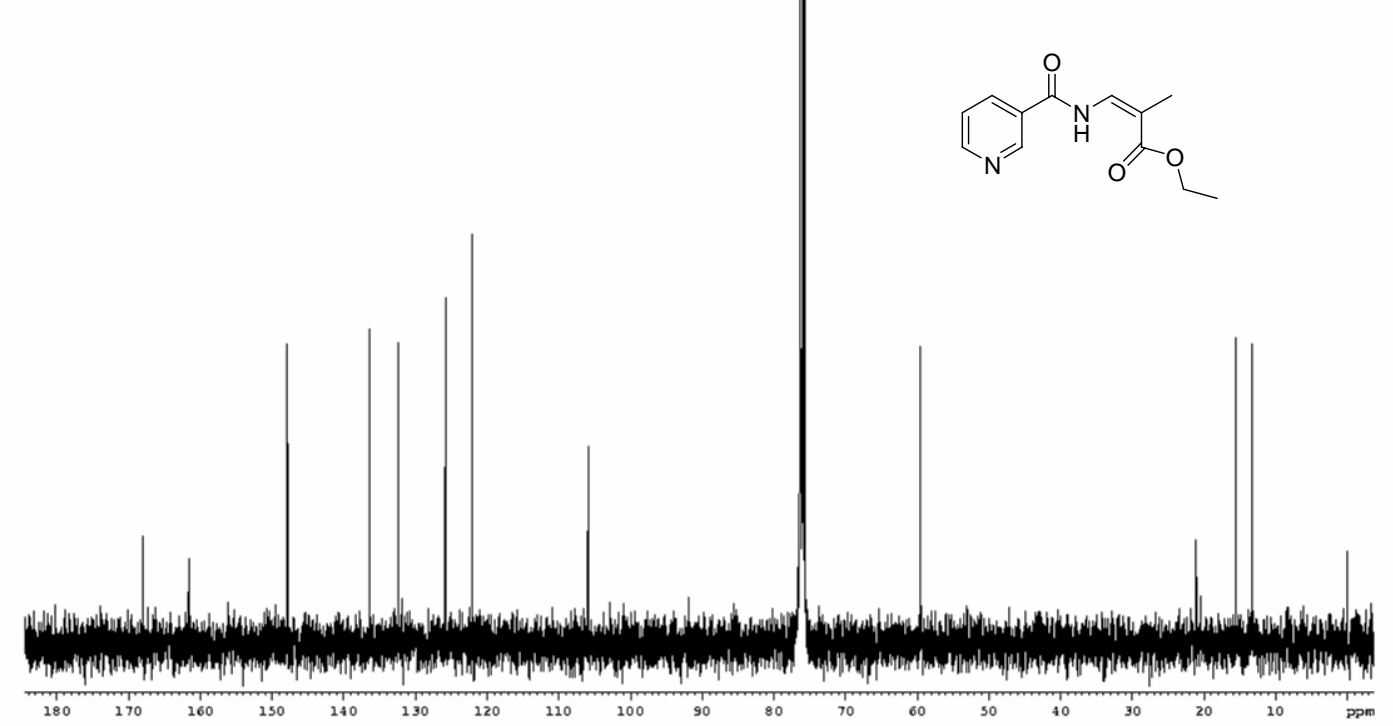


(E)-2-Methyl-3-[(pyridine-3-carbonyl)-amino]-acrylic acid ethyl ester, 14b (trans).

User - Matthew Vil1z

PROTOH MV2-88Bps 3 CDC13 L $^{3}$ matvil 26
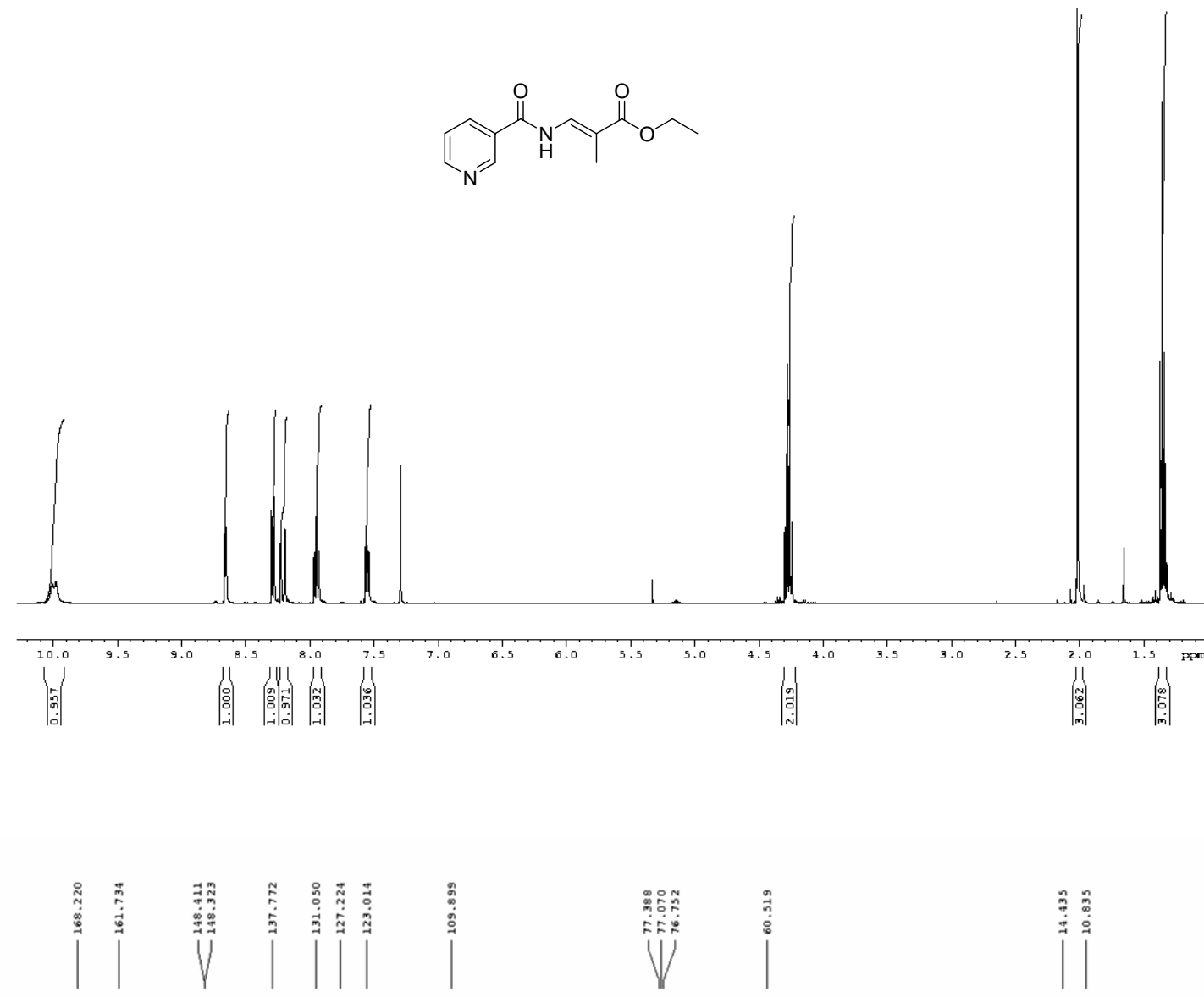

$\mid$

Usex - Matthew Villa

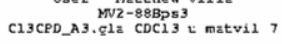

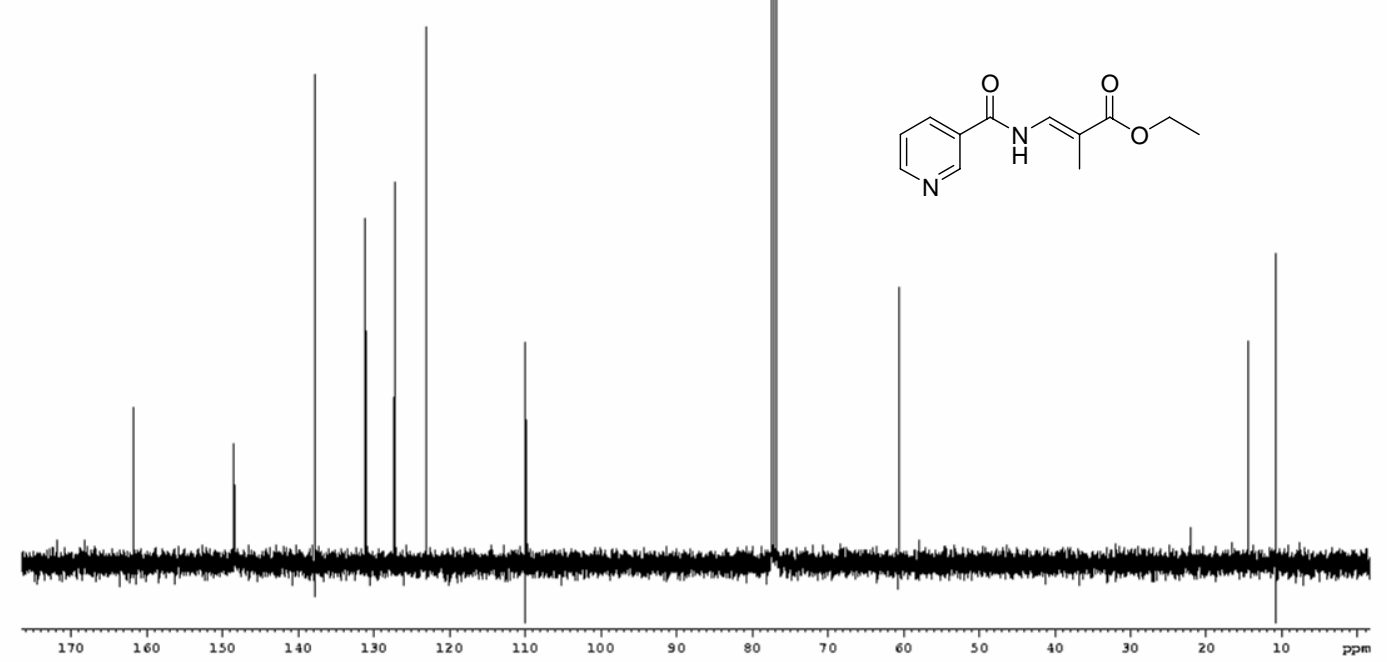


$N$-Formyl-2,2,5,5-(R)-tetramethyl-[1,3]dioxane-4-carboxylic acid amide, 12c.

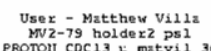
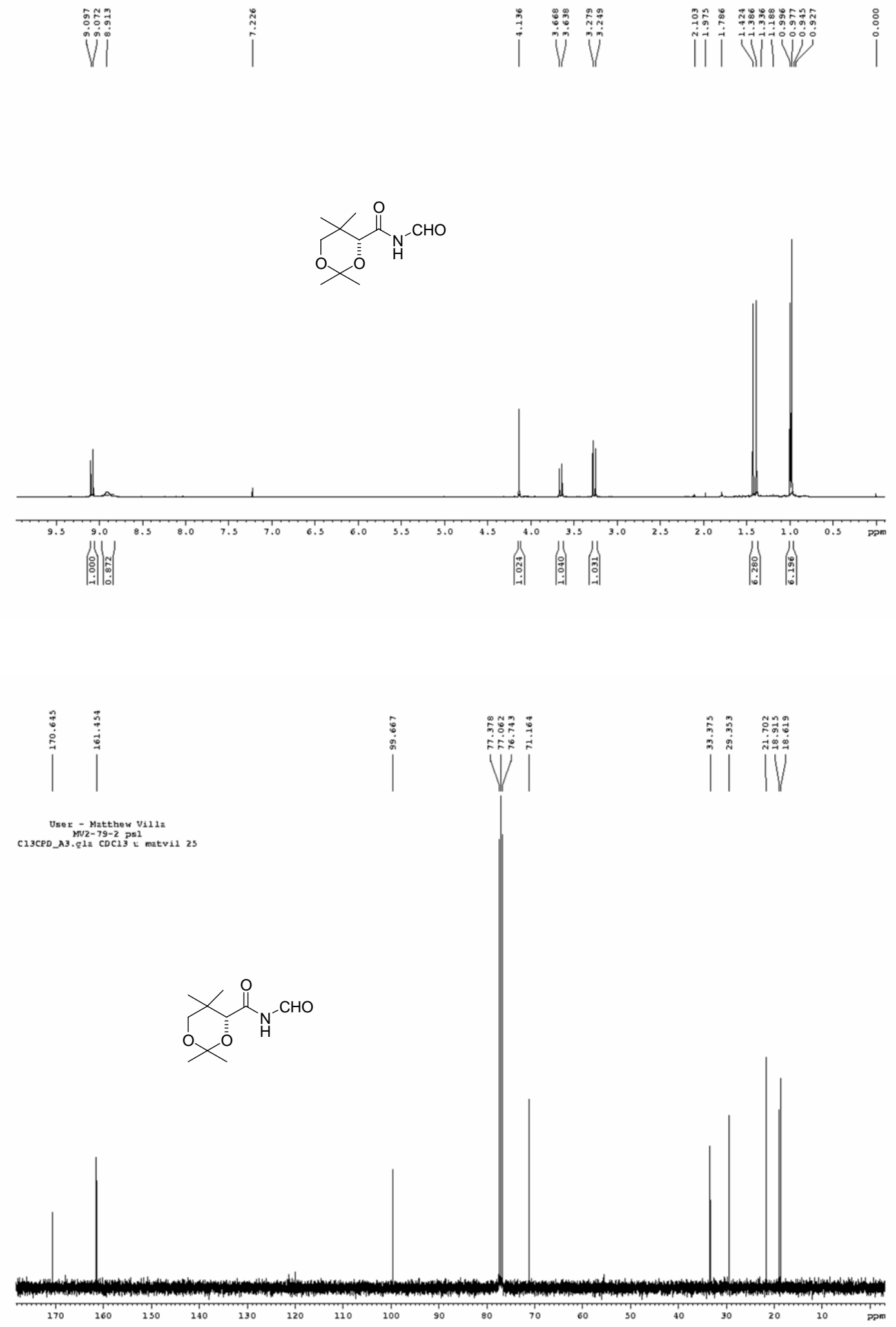
$(Z)-3-[((R)-2,2,5,5-T e t r a m e t h y l-[1,3]$ dioxane-4-carbonyl)-amino]-acrylic acid ethyl ester, 13c (cis).

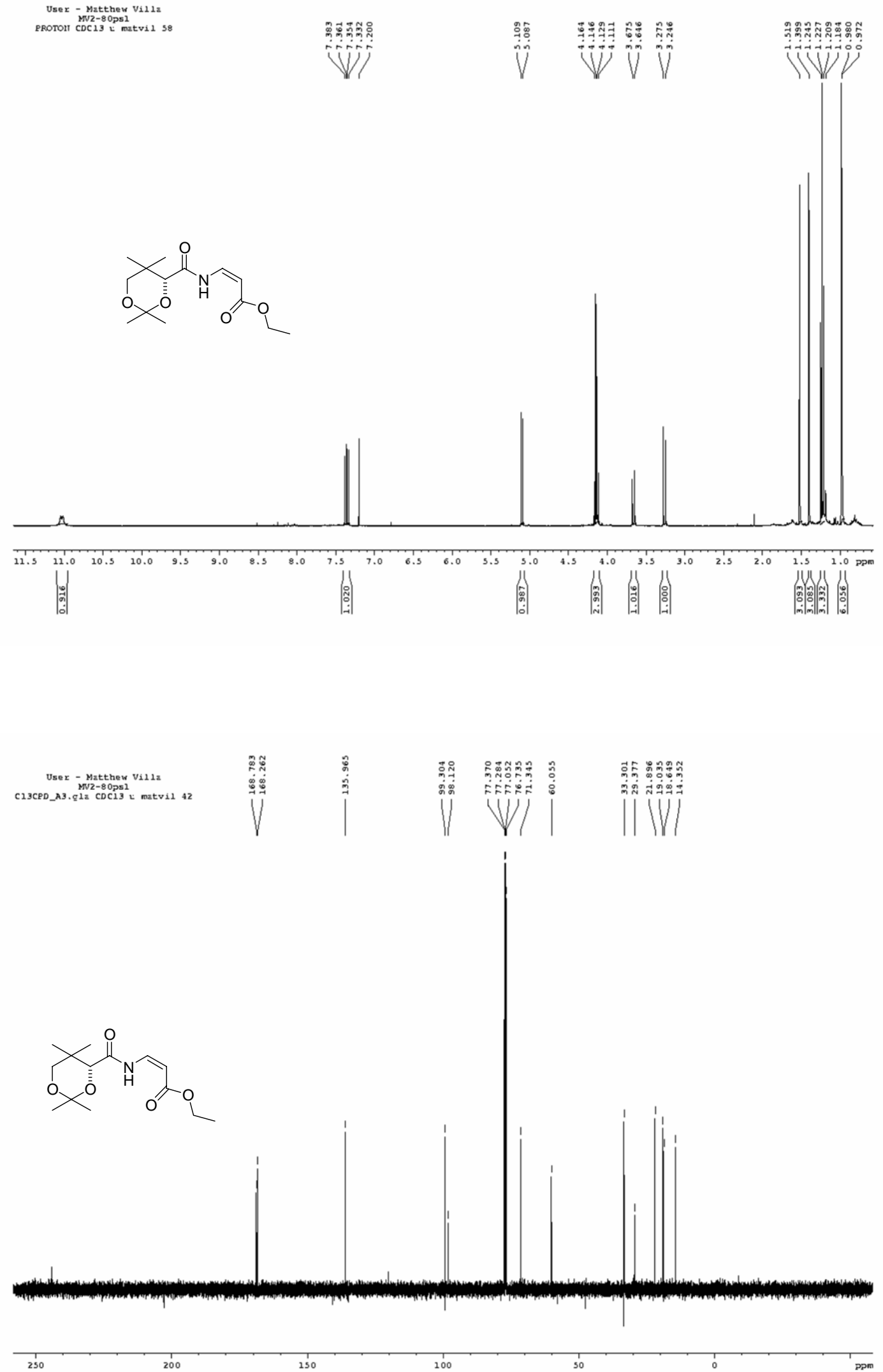


$(E)-3-[((R)-2,2,5,5-T e t r a m e t h y l-[1,3]$ dioxane-4-carbonyl)-amino]-acrylic acid ethyl ester, 13c (trans).
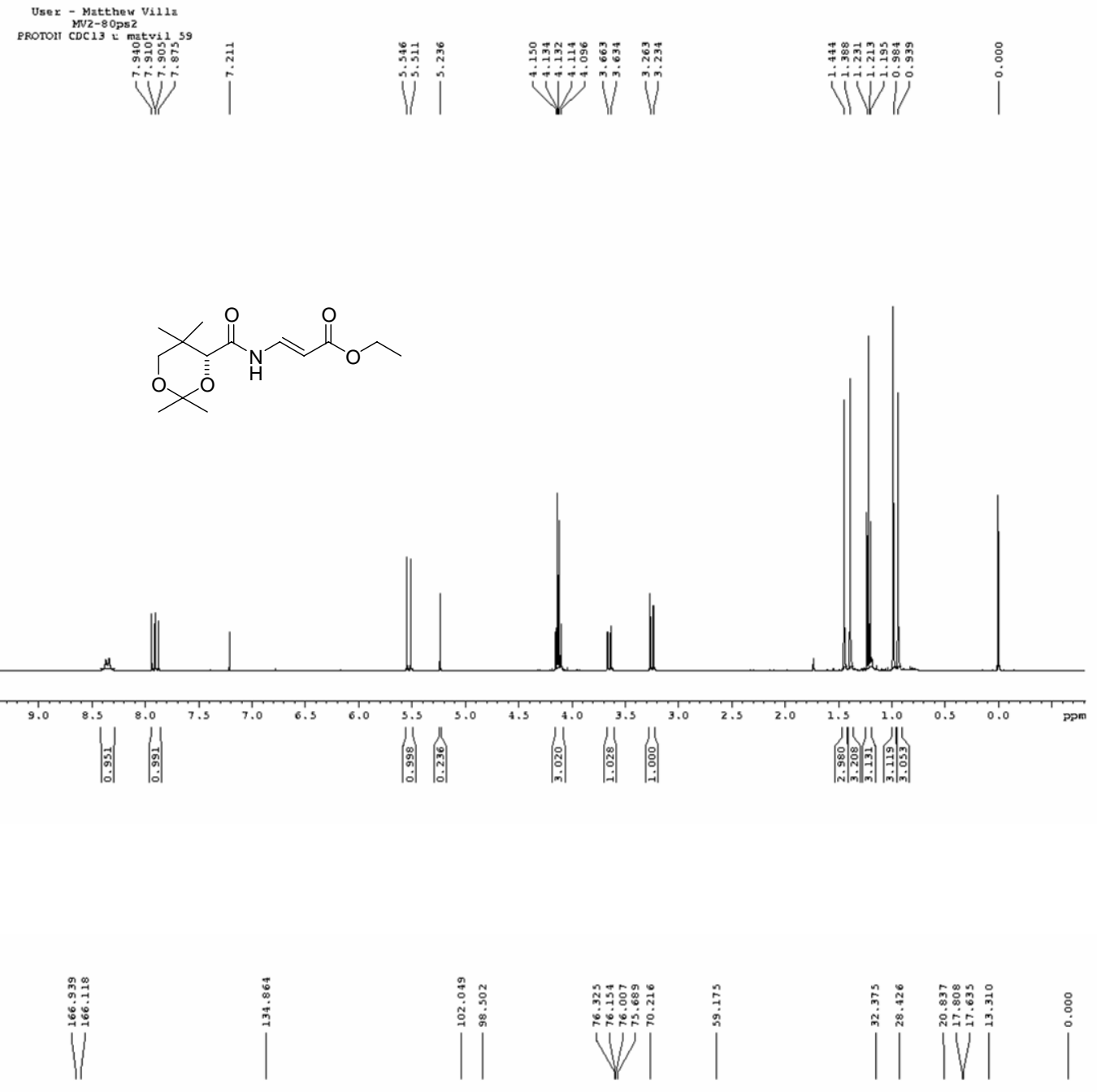

Usex - Matther Villa

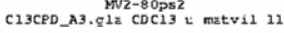

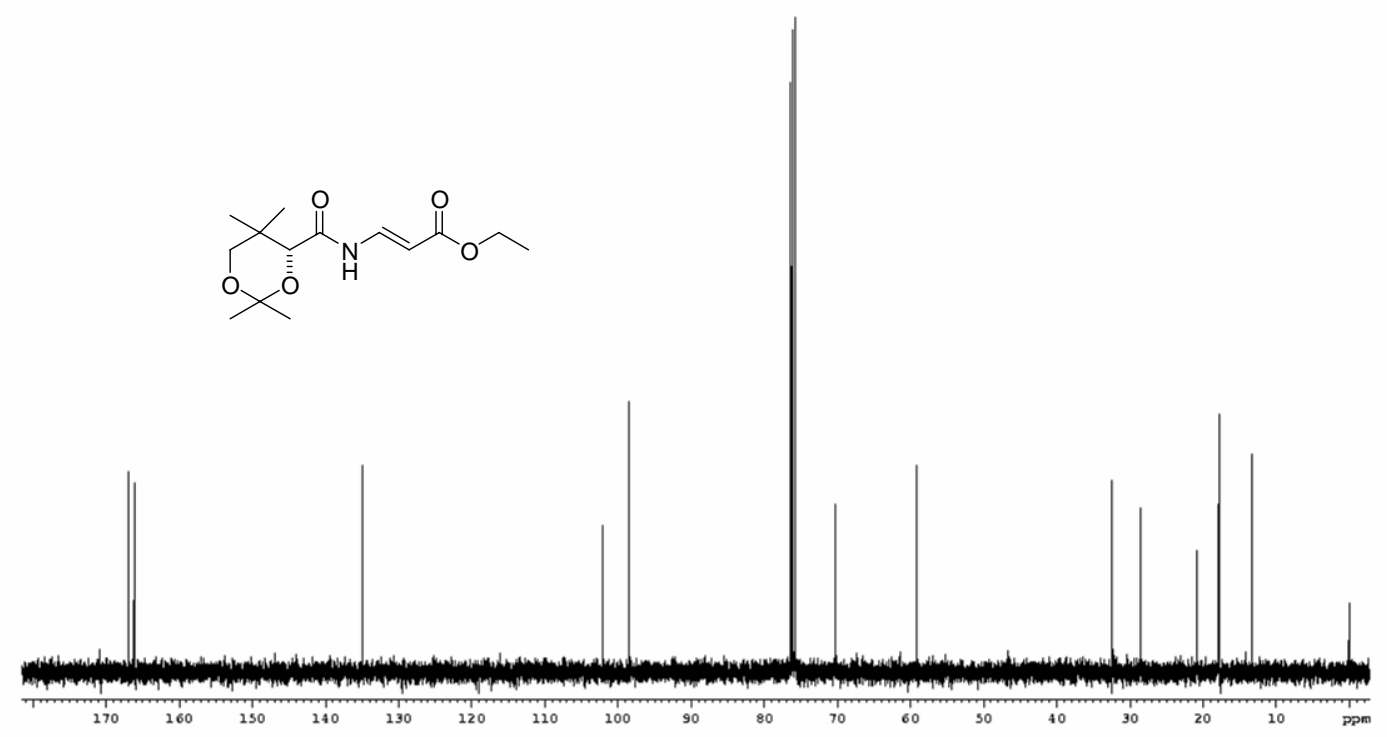


(Z)-2-Methyl-3-[((R)-2,2,5,5-tetramethyl-[1,3]dioxane-4-carbonyl)-amino]-acrylic acid ethyl ester, 14c (cis).

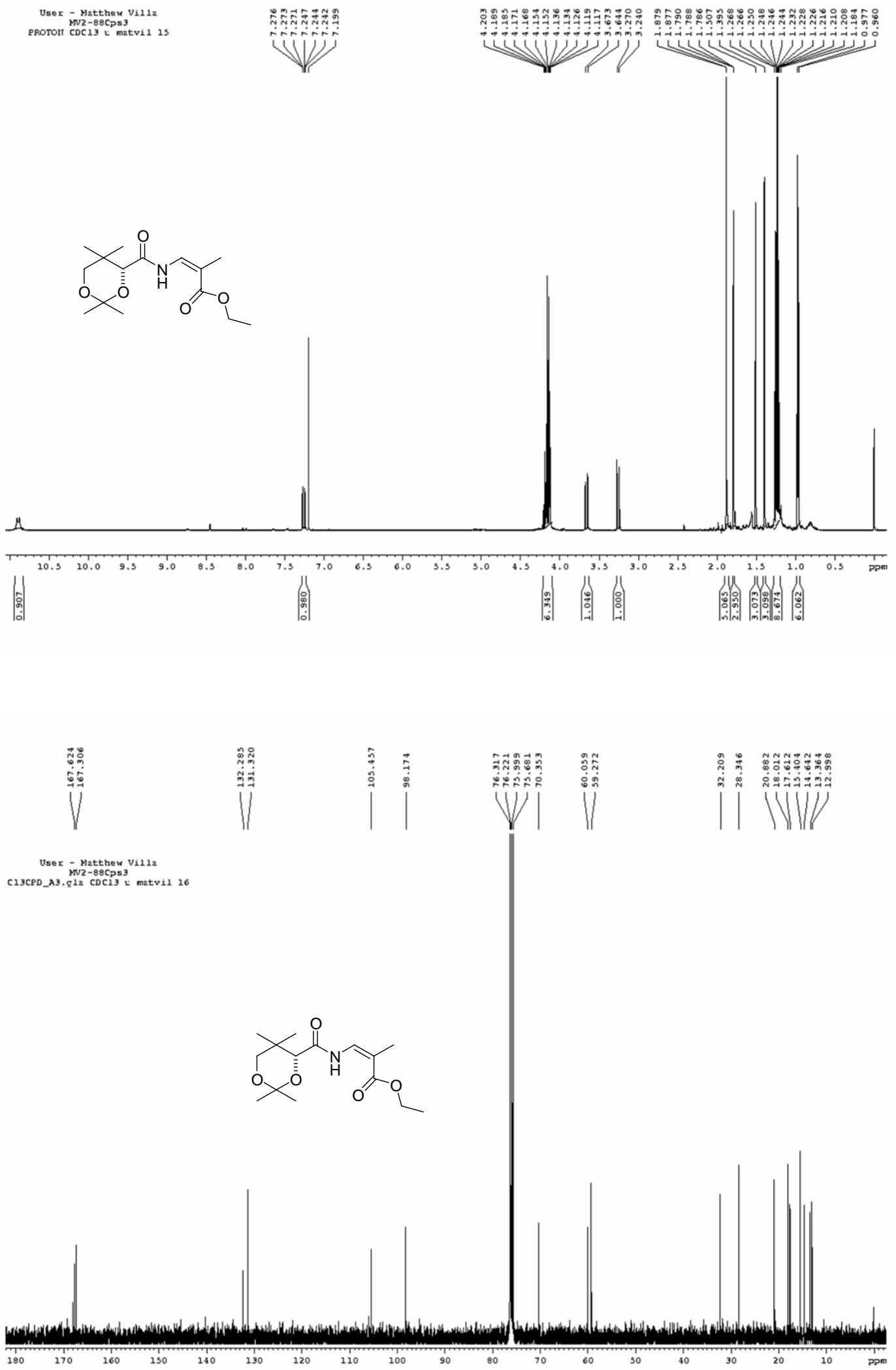


(E)-2-Methyl-3-[((R)-2,2,5,5-tetramethyl-[1,3]dioxane-4-carbonyl)-amino]-acrylic acid ethyl ester, 14c (trans).

Usex - Matther villa

MVI-88C ps4
PRoTOI CDC13 L matvil 31

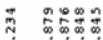

जi

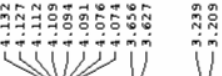

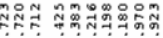

V V V ViVi
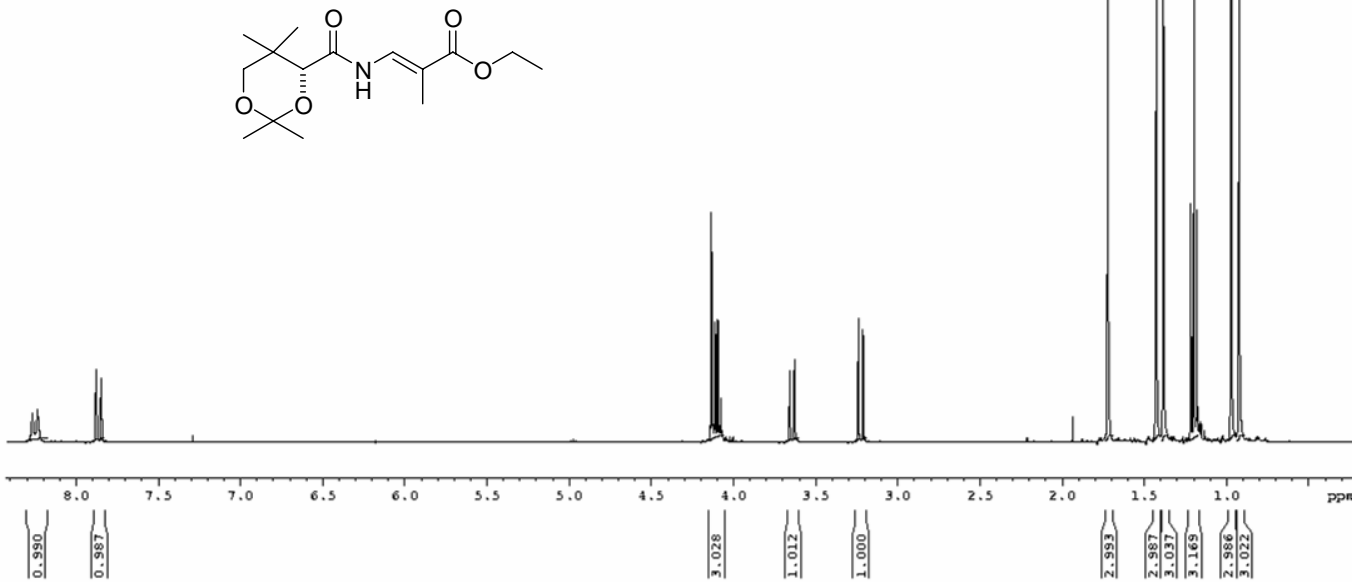

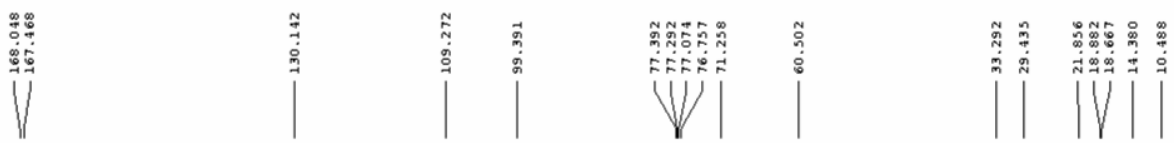

User - Matthew Villa

C13CPD_A3. Q1a CDC13 ti matvil \&

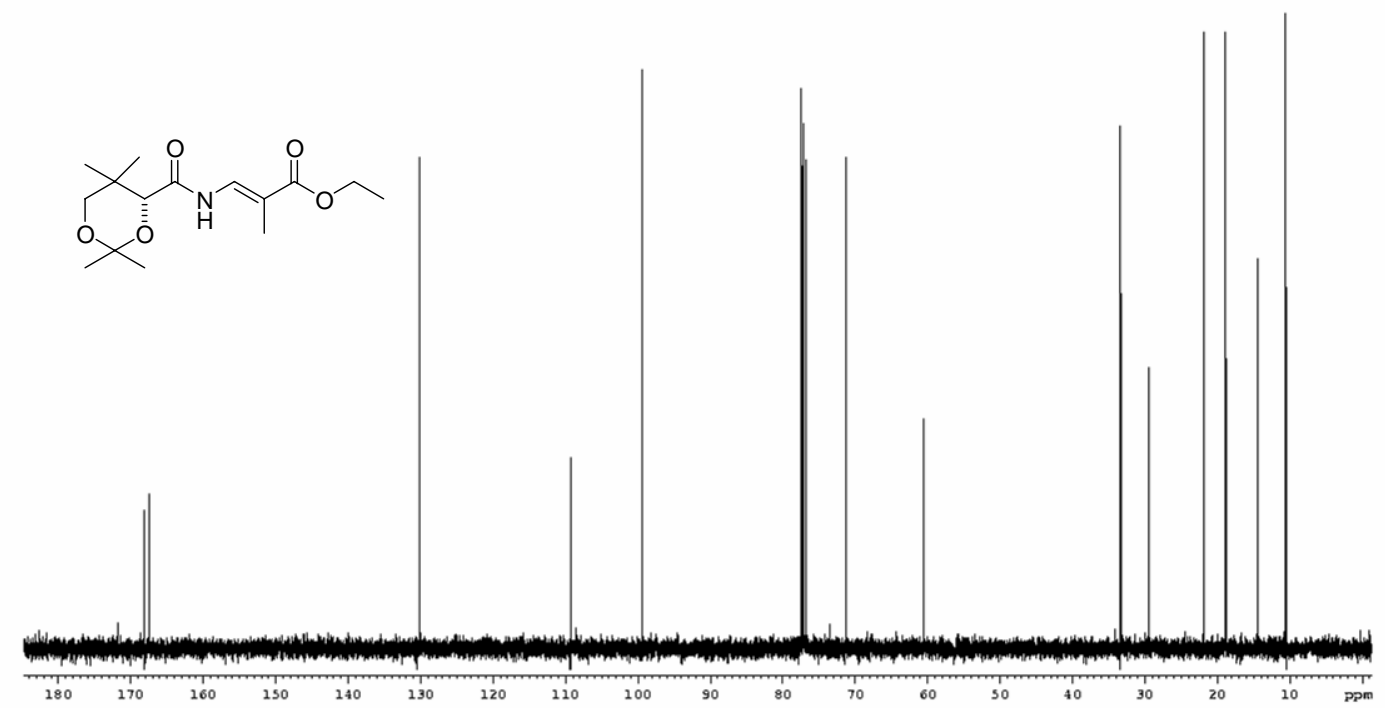

